Article

\title{
Gem-Quality Tourmaline from LCT Pegmatite in Adamello Massif, Central Southern Alps, Italy: An Investigation of Its Mineralogy, Crystallography and 3D Inclusions
}

\author{
Valeria Diella ${ }^{1, *(\mathbb{C}}$, , Federico Pezzotta ${ }^{2}$, Rosangela Bocchio ${ }^{3}$, Nicoletta Marinoni ${ }^{1,3}$, \\ Fernando Cámara ${ }^{3} \mathbb{D}$, Antonio Langone ${ }^{4}\left(\mathbb{D}\right.$, Ilaria Adamo ${ }^{5}$ and Gabriele Lanzafame ${ }^{6}(\mathbb{D}$ \\ 1 National Research Council, Institute for Dynamics of Environmental Processes (IDPA), Section of Milan, \\ 20133 Milan, Italy; nicoletta.marinoni@unimi.it \\ 2 Natural History Museum, 20121 Milan, Italy; fpezzotta@yahoo.com \\ 3 Department of Earth Sciences “Ardito Desio", University of Milan, 20133 Milan, Italy; \\ rosangela.bocchio@unimi.it (R.B.); fernando.camara@unimi.it (F.C.) \\ 4 National Research Council, Institute of Geosciences and Earth Resources (IGG), Section of Pavia, \\ 27100 Pavia, Italy; langone@igg.cnr.it \\ 5 Italian Gemmological Institute (IGI), 20123 Milan, Italy; ilaria.adamo@guest.unimi.it \\ 6 Elettra-Sincrotrone Trieste S.C.p.A., Basovizza, 34149 Trieste, Italy; gabriele.lanzafame@elettra.eu \\ * Correspondence: valeria.diella@cnr.it; Tel.: +39-02-50315621
}

Received: 12 November 2018; Accepted: 7 December 2018; Published: 13 December 2018

\begin{abstract}
In the early 2000s, an exceptional discovery of gem-quality multi-coloured tourmalines, hosted in Litium-Cesium-Tantalum (LCT) pegmatites, was made in the Adamello Massif, Italy. Gem-quality tourmalines had never been found before in the Alps, and this new pegmatitic deposit is of particular interest and worthy of a detailed characterization. We studied a suite of faceted samples by classical gemmological methods, and fragments were studied with Synchrotron X-ray computed micro-tomography, which evidenced the occurrence of inclusions, cracks and voids. Electron Microprobe combined with Laser Ablation analyses were performed to determine major, minor and trace element contents. Selected samples were analysed by single crystal $X$-ray diffraction method. The specimens range in colour from colourless to yellow, pink, orange, light blue, green, amber, brownish-pink, purple and black. Chemically, the tourmalines range from fluor-elbaite to fluor-liddicoatite and rossmanite: these chemical changes occur in the same sample and affect the colour. Rare Earth Elements (REE) vary from 30 to 130 ppm with steep Light Rare Earth Elemts (LREE)-enriched patterns and a negative Eu-anomaly. Structural data confirmed the elbaitic composition and showed that high manganese content may induce the local static disorder at the $\mathrm{O}(1)$ anion site, coordinating the $\mathrm{Y}$ cation sites occupied, on average, by $\mathrm{Li}, \mathrm{Al}$ and $\mathrm{Mn}^{2+}$ in equal proportions, confirming previous findings. In addition to the gemmological value, the crystal-chemical studies of tourmalines are unanimously considered to be a sensitive recorder of the geological processes leading to their formation, and therefore, this study may contribute to understanding the evolution of the pegmatites related to the intrusion of the Adamello pluton.
\end{abstract}

Keywords: granitic pegmatite; gem-quality tourmaline; Adamello Massif; Central Alps; Italy

\section{Introduction}

"Tourmaline" is considered one of the most beautiful gemstones, because it occurs in a large spectrum of colours, as well as in multi-coloured crystals [1]. In Italy, gem-quality tourmaline is 
known to be from the historic locality, at present rather exhausted, of Elba Island (Tyrrhenian sea), from which it derived the root-name elbaite, given to Li-bearing and sodium-rich tourmalines of the alkali group [2]. For a very long time, Elba Island has remained the only known locality providing gem-quality tourmaline crystals in Italy. Nevertheless, since 2001, an additional significant locality has been discovered and described in the Italian Alps, which is the Adamé valley, along the western border of the late Alpine Adamello tonalite massif, in the central Southern Alps [3].

In the Adamé valley, gem-quality tourmaline occurs in multi-coloured elongated crystals up to $7 \mathrm{~cm}$ in length in the miarolitic cavities of LCT pegmatite (i.e., a pegmatite with prevailing minerals of lithium, caesium and tantalum) hosted in contact with metamorphic sandstones, belonging to a Permian-Mesozoic sedimentary sequence. The locality is included in the Adamello Park natural reservation. The miarolitic pegmatite has been found on the steep slope of Forcel Rosso pass, disrupted in large blocks in an ancient landslide, at an altitude of about $2600 \mathrm{~m}$. Tourmaline group minerals occur together with albite, smoky quartz, K-feldspar, mica (lepidolite-muscovite) and other accessory minerals such as fluorapatite, fluorite, and several $\mathrm{Nb}-\mathrm{Ta}-\mathrm{Sb}$ oxides, all very interesting from a scientific and collector point of view. In consideration of the significant potential of the pegmatite for mineral production, the locality underwent, under strict protection, an official detailed field investigation for scientific purposes, with the supervision of one of the authors (F.P.). The first results [3] show that the tourmaline crystals have a zoned composition, mostly characterized by fluor-elbaite, with fluor-liddicoatite developing at the antilogous pole of the crystals. Colours range from colourless to yellow, pink, orange, light blue, green, amber, brownish-pink, purple and black (Figure 1). Gem-quality tourmalines are present as sectors of larger crystals, and rough fragments can reach a maximum weight of $2-3 \mathrm{~g}$.

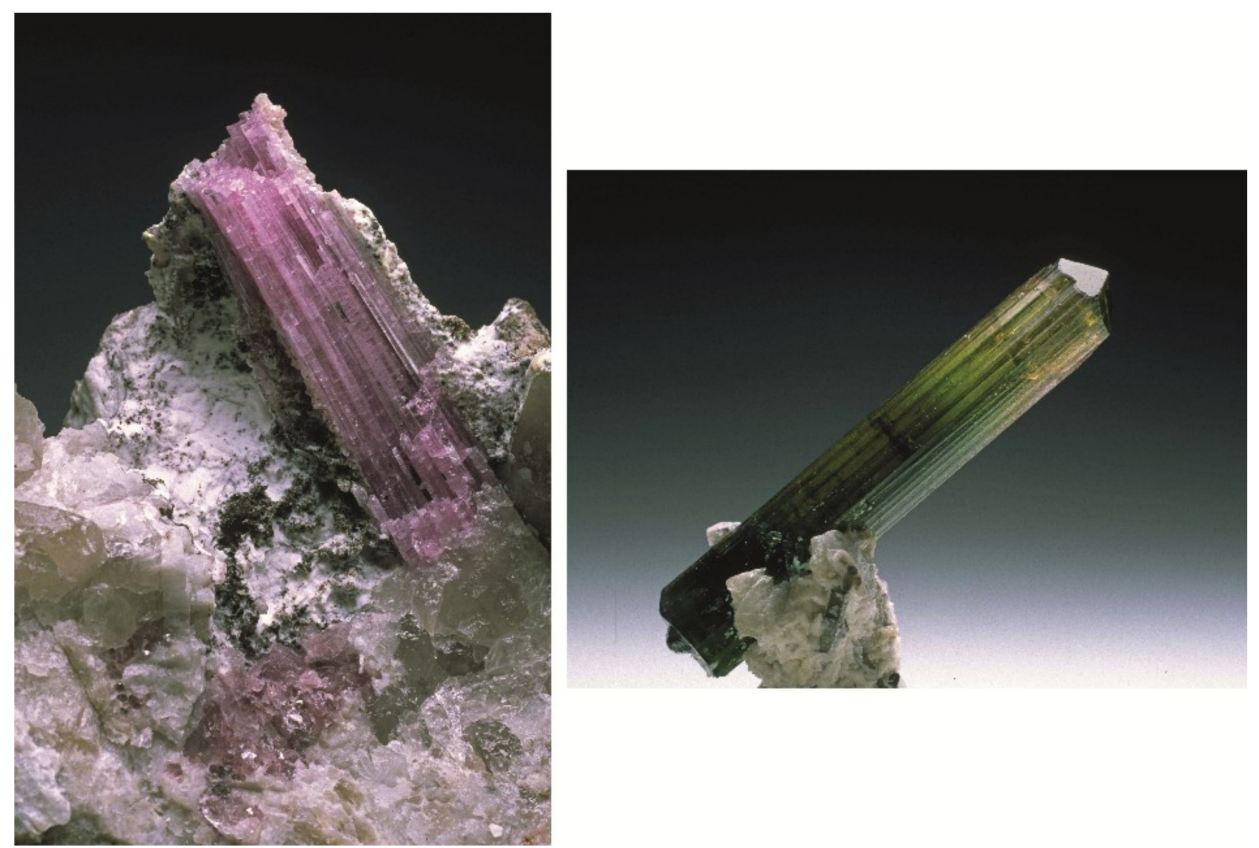

Figure 1. Crystals of pink ( $3.5 \mathrm{~cm}$ long) and green $(5 \mathrm{~cm}$ long) tourmalines from Valle Adamé, Adamello Massif. Natural History Museum of Milan collection. Photos by R. Appiani.

The collected materials are preserved in the mineralogical collections of the Museum of Natural History of Milan and at the Museum of the Adamello Natural Park. These gem tourmalines have not entered the market, because the deposit is located within the protected areas of the Adamello Natural Park and collecting is allowed only for scientific purposes. At present, only a few gems have been faceted (Figure 2). 

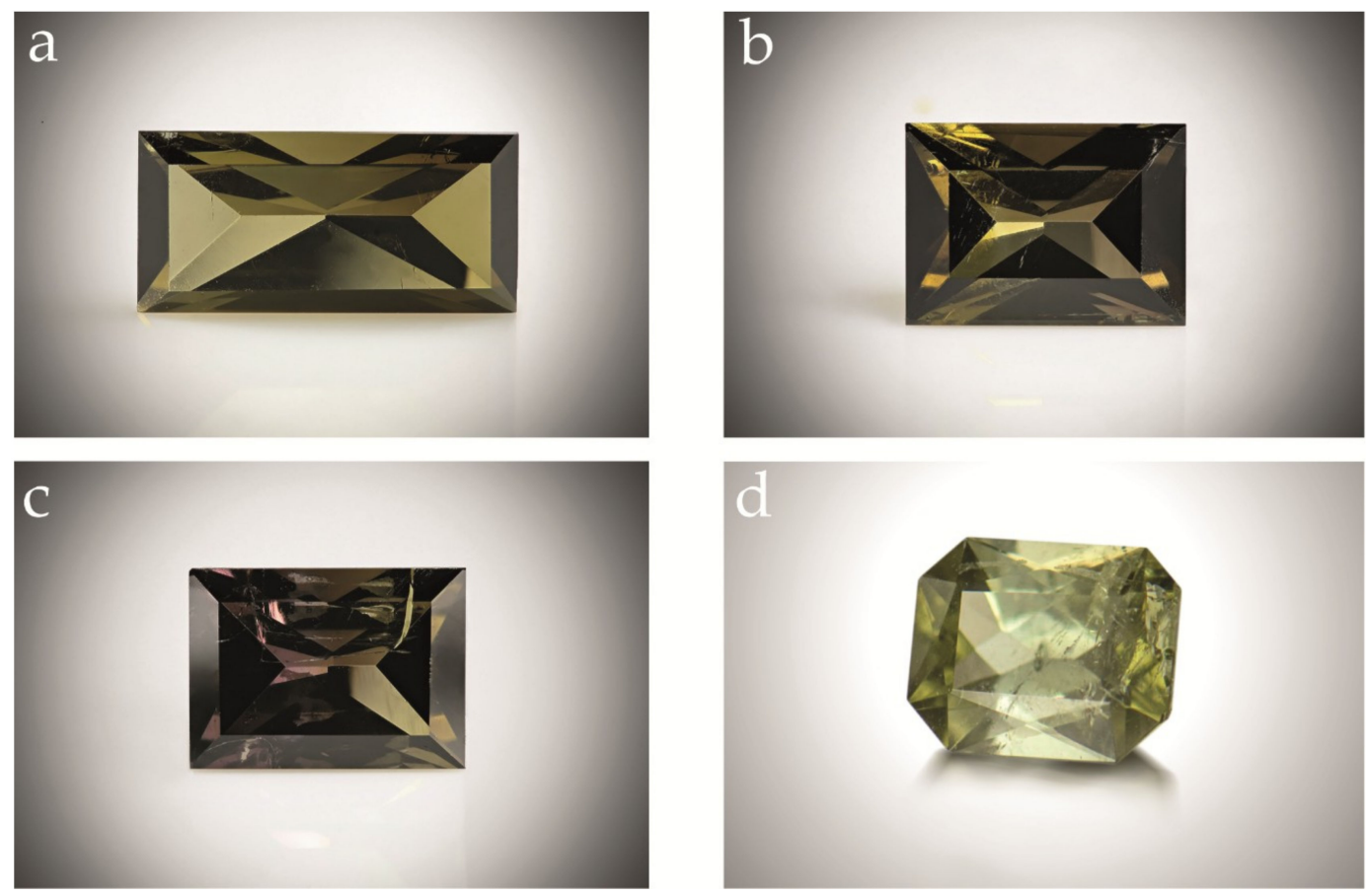

Figure 2. Cut tourmalines analysed in this study. (a) $3.97 \times 8.31 \times 2.61 \mathrm{~mm}$; (b) $4.73 \times 6.58 \times 3.35 \mathrm{~mm}$; (c) $4.61 \times 6.36 \times 3.26 \mathrm{~mm}$, (d) $5.72 \times 7.16 \times 4.03 \mathrm{~mm}$, corresponding to samples 13, 14, 15 and 16 described below in Table 1. Photos by M. Chinellato and F. Picciani.

As mentioned above, tourmaline represents one of the most beautiful gemstones and may be characterized through multi-methodological methods including non-invasive and non-destructive techniques, such as Fourier Transform Infrared Spectroscopy and Raman Spectroscopy, when a conservative treatment of sample is required ([4] and references therein). Many studies recognize a significant petrological interest of this mineral, as it can contribute to the reconstruction of the evolution of the history of a crystalline basement, e.g., [5].

In view of this, and considering that the Adamello Massif is one of the most geologically studied portions of the Alpine chain, our study aims to provide a complete mineralogical characterization of these recently discovered gem tourmalines, as well as an interesting contribution to the knowledge of local pegmatite mineralization. We investigated a suite of cut and rough samples selected from the mineralogical collections of the Natural History Museum of Milan, through gemmological analyses, electron microprobe chemical analyses (EPMA) and laser ablation-inductively coupled plasma-mass spectrometry (LA-ICP-MS). The unit cell parameters and refinement of crystal structure of three selected tourmalines were carried out by the single-crystal X-ray diffraction method. Selected rough samples have also been examined by means of synchrotron X-ray computed micro-tomography $(\mathrm{X}-\mu \mathrm{CT})$, which made it possible to obtain a 3D visualisation of the inner objects (pore, inclusions, etc.) within a volume [6,7]. This technique does not require any sample preparation and makes it possible to overcome the problem of deriving the 3D results from the traditional 2D data obtained by traditional imaging techniques such as optical and scanning electron microscopy. In the present study, a fully characterisation of inclusions in terms of size, shape and orientation was performed.

\section{Background Information}

Tourmaline is a ring-silicate crystallizing in the acentric $3 m$ point group (ditrigonal pyramidal) with the $R 3 m$ space group and a general formula of $X_{3} Y_{6} \mathrm{Z}_{6} \mathrm{~T}_{6} \mathrm{O}_{18}\left(\mathrm{BO}_{3}\right)_{3} V_{3} \mathrm{~W}$ [8]. The unit cell consists of a six-fold ring of tetrahedra ( $T$ sites, occupied primarily by $\mathrm{Si}$ ) on top of a concentric arrangement of three $Y$-site and six $Z$-site octahedra. The $X$ site is nine-coordinated and situated centred over of the six-fold ring and can be occupied by $\mathrm{Na}^{+}, \mathrm{Ca}^{2+}, \mathrm{K}^{+}$cations or be vacant. The $Y$ and $Z$ sites may 
contain a large variety of cations such as $\mathrm{Mg}^{2+}, \mathrm{Fe}^{2+}, \mathrm{Fe}^{3+}, \mathrm{Li}^{+}, \mathrm{Al}^{3+}, \mathrm{Mn}^{2+}, \mathrm{Cu}^{2+}, \mathrm{Ti}^{4+}, \mathrm{Cr}^{3+}, \mathrm{V}^{3+}$ and $\mathrm{Al}^{3+}, \mathrm{Mg}^{2+}, \mathrm{Fe}^{2+}, \mathrm{Fe}^{3+}, \mathrm{Cr}^{3+}, \mathrm{V}^{3+}$, respectively. Three planar triangular boron-centred polyhedra $\left(\mathrm{BO}_{3}\right)$ are further present, which are roughly coplanar with the groups of octahedra in the unit cell, and roughly perpendicular to the $c$ axis. The $V$ and $W$ sites are anion sites that are occupied by $\mathrm{OH}^{-}$ or $\mathrm{O}^{2-}$ (or both) at the $V$ site, and $\mathrm{OH}^{-}, \mathrm{F}^{-}$, or $\mathrm{O}^{2-}$ at the $W$ site. The $\mathrm{Cl}$ contents are generally negligible. Given the range of elements and heterovalent substitutions that can be accommodated by the tourmaline structure, the high number of different end-member compositions constitute a super group consisting of quite a large number of species, as approved by the International Mineralogical Association's Commission on New Minerals, Nomenclature and Classification [2,9-11].

\section{Geological Setting}

The Tertiary Adamello batholith outcrops over an area of ca. $670 \mathrm{~km}^{2}$ in the Central Southern Alps (Northern Italy) (CNR, Italian Geological Map Adamello-Presanella, 1:50,000). It consists of four composite plutons decreasing in age from south (ca. $42 \mathrm{Ma}$ ) to north (ca. $31 \mathrm{Ma}$ ) and is composed mainly of granitoid rocks (granodiorite, tonalite, quartz diorite) with minor amounts of diorite and gabbro [12-14]. The plutons intrude into the South-Alpine crystalline basement and its Permo-Mesozoic sedimentary cover (Figure 3). The studied tourmaline crystals were collected in pegmatite dykes occurring in the thermo-metamorphic contact aureole between the igneous rocks and the surrounding sediments, at the northern slope of the Foppa mountain $(2752 \mathrm{~m}$ ) in a gully of Forcel Rosso (or "Vallone del Forcel Rosso"').

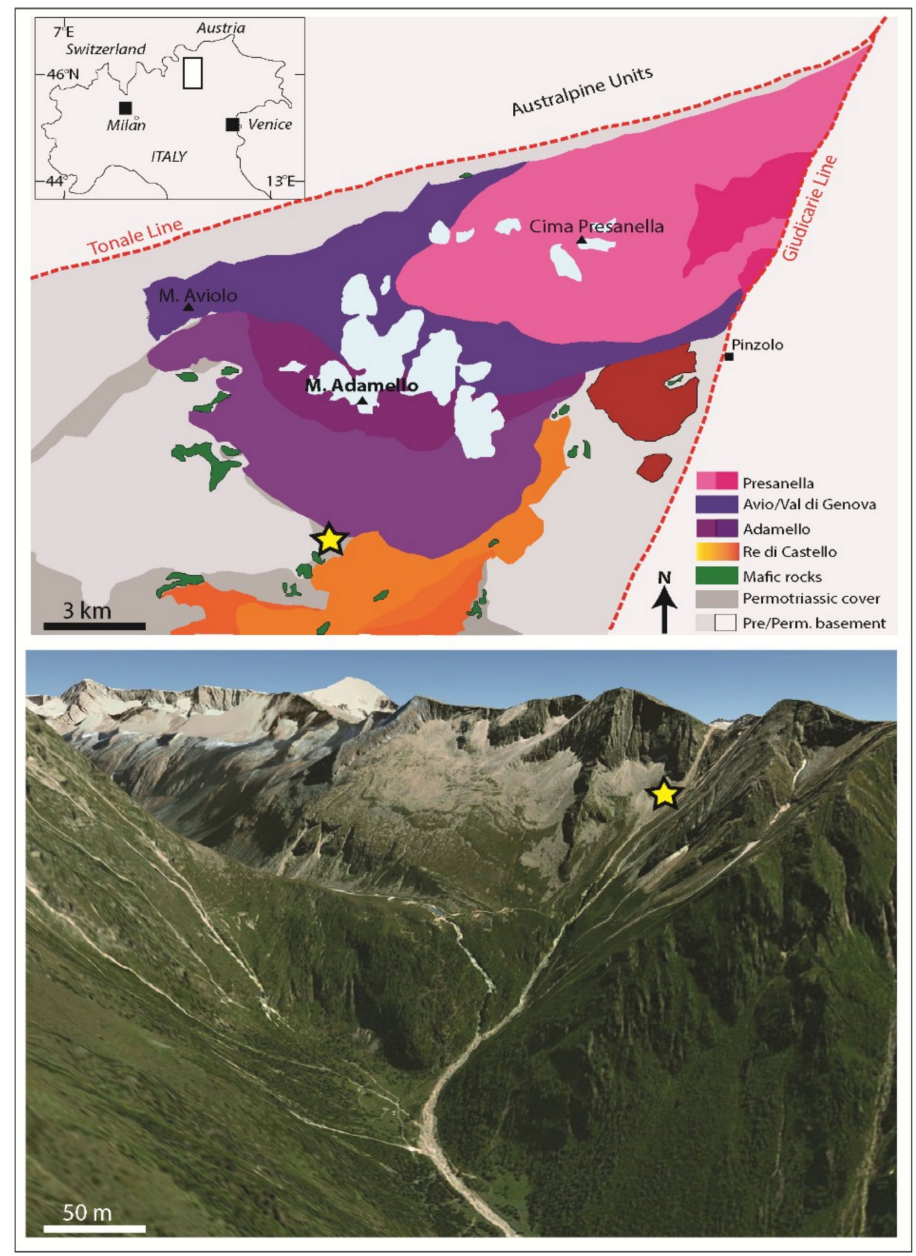

Figure 3. Geological sketch map of the Adamello pluton and a view of the area of Forcel Rosso. Photo by F. Pezzotta. The stars indicate the location of the studied pegmatite dikes. 
The pegmatites are sub-horizontal and hosted in meta-sandstones enriched in accessory tourmaline. The larger veins are up to approximately $1.5 \mathrm{~m}$ in thickness, and some tens of meters long. Despite their relatively small size, these pegmatites display a quite strong asymmetric zoning with a layered lower unit composed by fine to medium grain size assemblage of feldspars and quartz, with accessory muscovitic mica, schorl and spessartine, and an upper coarse-grained unit composed of an assemblage of quartz and feldspars, rich in schorl and muscovitic mica, occasionally displaying cores with milky white quartz masses, lepidolite overgrowth at the rim of muscovite blades, granular lepidolite masses and multi-coloured tourmalines. Miarolitic cavities are not common, but they can be locally abundant, with a diameter achieving several decimetres in length, at the coarse-grained cores of the veins.

Accessory minerals in cavities include pink to green fluor-apatite, purple and pale green fluorite, late-stage calcite, several pyrochlore group minerals and various $\mathrm{Ta}-\mathrm{Nb}$ oxides [3].

\section{Materials and Methods}

Sixteen samples of tourmaline from different miarolitic cavities were selected for this study, and they are described in Table 1.

Table 1. Description of the studied tourmalines.

\begin{tabular}{|c|c|c|c|c|}
\hline Sample & $\begin{array}{c}\text { Cavity } \\
\text { Name/Description * }\end{array}$ & Colour & \multicolumn{2}{|c|}{ Analytical Techniques } \\
\hline 1 & Cavity 1 & Pink & $\begin{array}{l}\text { Electron microprobe } \\
\text { (WDS) }\left(\left(^{\circ}\right)\right.\end{array}$ & Single crystal XRD $\left(^{\circ}\right)$ \\
\hline 2 & Cavity boulder 2 & Light blue & $\begin{array}{l}\text { Electron microprobe } \\
\text { (WDS) }\left({ }^{\circ}\right)\end{array}$ & Single crystal XRD $\left(^{\circ}\right)$ \\
\hline 3 & Pizio cavity & $\begin{array}{l}\text { Colourless to } \\
\text { brown }\end{array}$ & $\begin{array}{l}\text { Electron microprobe } \\
\text { (WDS) }\left({ }^{\circ}\right)\end{array}$ & \\
\hline $\begin{array}{l}\text { 4: sample cut perpendicular } \\
\text { to the } c \text {-axis }\end{array}$ & Pizio cavity & Pink green & $\begin{array}{l}\text { Electron microprobe } \\
\text { (WDS) }\left({ }^{\circ}\right)\end{array}$ & \\
\hline 5 & Cavity 3 & Light green yellow & $\begin{array}{l}\text { Electron microprobe } \\
\text { (WDS) }\left(\left(^{\circ}\right)\right.\end{array}$ & \\
\hline 6 & Cavity 4 & $\begin{array}{l}\text { Yellow to } \\
\text { colourless }\end{array}$ & $\begin{array}{l}\text { Electron microprobe } \\
\text { (WDS) }\left(\left(^{\circ}\right)\right.\end{array}$ & \\
\hline 8 & Cavity 6 & & & $\begin{array}{c}\text { Synchrotron X-ray } \\
\text { computed } \mu \text {-tomography }\end{array}$ \\
\hline 9 & "Black quartz" cavity & Pink green & $\begin{array}{l}\text { Electron microprobe } \\
\text { (WDS) }\left(\left(^{\circ}\right)\right.\end{array}$ & \\
\hline $\begin{array}{l}\text { 10: double } \\
\text { terminated sample }\end{array}$ & Inv.\# M36742 cavity & Browm green & $\begin{array}{l}\text { Electron microprobe } \\
\text { (WDS) }\left(\left(^{\circ}\right)\right.\end{array}$ & \\
\hline $\begin{array}{l}\text { 11: homologous } \\
\text { terminated sample }\end{array}$ & Inv.\# M36742 cavity & Green to colourless & $\begin{array}{l}\text { Electron microprobe } \\
(\text { WDS })\left({ }^{\circ}\right)\end{array}$ & \\
\hline $\begin{array}{l}\text { 12: sample cut } \\
\text { perpendicular to the } c \text {-axis }\end{array}$ & Inv.\# M36742 cavity & Blue & $\begin{array}{l}\text { Electron microprobe } \\
(\text { WDS })\left({ }^{\circ}\right)\end{array}$ & Single crystal XRD $\left(^{\circ}\right)$ \\
\hline 16 & $\begin{array}{l}\text { Cut stone }(1.225 \mathrm{ct}) \\
\text { from pocket }\end{array}$ & Pale green & Specific gravity & Refractive index \\
\hline
\end{tabular}

* Cavity numbers refer to the unpublished field note-book made during the collecting campaigns performed by the Natural History Museum of Milan in 2001 and 2002. The reported inventory numbers are the ones given to specimens catalogued in the Mineralogical Collections of the Natural History Museum of Milan. ${ }^{\circ}$ Wavelength Dispersive Spectroscopy (WDS); X-ray Diffractometry (XRD). 
Four faceted samples (13-16) were examined by standard gemmological methods at the Italian Gemmological Institute in Milan in order to determine their refractive index, hydrostatic specific gravity and microscopic features. The refractive indices were measured with a Krüss refractometer (A. Krüss Optronic, Hamburg, Germany) using ordinary light source with a sodium filter ( $589 \mathrm{~nm}$ ) and a methylene iodide as a contact liquid $(n=1.80)$. A Mettler hydrostatic balance was used to determine the specific gravity in bi-distilled water.

Two grains (7 and 8 ) were used for synchrotron X-ray computed micro-tomography. The samples, with a size of about $1.5 \mathrm{~mm}$, were imaged at the SYRMEP beamline of ELETTRA synchrotron facility (Trieste, Italy). The computer micro-tomography experiments were performed using a polychromatic X-ray beam (white beam mode. With this configuration, the outcome beam from the storage ring was intercepted before the monochromator and pre-filtered with $1.5 \mathrm{~mm}$ of $\mathrm{Si}$ and $1.0 \mathrm{~mm}$ of $\mathrm{Al}$. A water-cooled, 16 bit, $2028 \times 2048$ pixel microscope Charge-Coupled Device (CCD) camera, coupled with a 100 micron thick LuAG scintillator screen was used as detector. The mean energy was set to $28 \mathrm{keV}$. Pixel size was set at $2 \mu \mathrm{m} /$ pixel, yielding a field of view of $5 \times 5 \mathrm{~mm}$.

The software suite Syrmep Tomo Project (STP) $[15,16]$ was used to reconstruct two-dimensional axial slices from the sample projections, applying the filtered back projection algorithm $[17,18]$. Before image reconstruction, a single-distance phase retrieval algorithm was applied to the projection images [19] using the STP software and setting the $\delta / \beta$ ratio to 15 (see $[15,16]$ for details about the phase retrieval process).

Ten of the selected fragments, 1-6 and 9-12, were embedded in epoxy resin, polished and prepared for EMPA and LA-ICP-MS analysis. Optical images of the samples were performed in advance using a stereo microscope Leica M205 C in reflected light. The backscattered electron images and quantitative chemical analyses of major and minor elements were obtained with the JEOL JXA-8200 electron microprobe in wavelength dispersion mode (EMPA-WDS) at the laboratory of the Department of Earth Sciences of the University of Milan under the following conditions: $15 \mathrm{kV}$ accelerating voltage, $5 \mathrm{nA}$ beam current, and a count time of $60 \mathrm{~s}$ on peak and $30 \mathrm{~s}$ on the background, with a $1 \mu \mathrm{m}$ diameter beam. The $\mathrm{K} \alpha$ wavelengths and natural standards of pure metals were used for calibration: $\mathrm{F}$ (hornblende), $\mathrm{Ti}$ (ilmenite), Mn and Zn (rhodonite), K (K-feldspar), Na (omphacite), Fe (fayalite), Ca, Si and Al (grossular), $\mathrm{Mg}$ (olivine), $\mathrm{Cr}$ and $\mathrm{V}$ (pure elements). The raw data were corrected for matrix effects using a conventional $\Phi \rho Z$ routine in the JEOL software package. Both Fe and Mn were calculated as $\mathrm{Fe}^{2+}$ and $\mathrm{Mn}^{2+}$. The structural formula was calculated on the basis of 31 anions $(\mathrm{O}, \mathrm{OH}$ and $\mathrm{F})$, and $\mathrm{Li}_{2} \mathrm{O}, \mathrm{B}_{2} \mathrm{O}_{3}$ and $\mathrm{H}_{2} \mathrm{O}$ were calculated based on the assumed elbaite stoichiometry.

Rare earth and selected trace elements were determined by laser ablation-inductively coupled plasma-mass spectroscopy (LA-ICP-MS) at the IGG-CNR Laboratory of Pavia. The instrument consisted of a Quantel Brilliant $213 \mathrm{~nm}$ Nd:YAG laser (New Wave Research) coupled to a Perkin Elmer DRCe quadrupole ICPMS. The spot size was $55 \mu \mathrm{m}$, using NIST SRM 610 glass as an external standard and Si as an internal standard, as analysed by microprobe. Precision and accuracy estimated on the basaltic glass standard BCR2 and NIST612 were better than $10 \%$.

Three fragments $(\sim 500 \mu \mathrm{m})$ from samples 1,2 , and 12 were cut for single crystal X-ray diffraction measurements. Data were collected with an Xcalibur-Oxford Diffraction diffractometer (Oxford Diffraction Ltd., Abingdon, UK) equipped with a CCD, using graphite-monochromatized MoK $\alpha$ radiation and operated at $50 \mathrm{kV}$ and $30 \mathrm{~mA}$. To maximize the reciprocal space coverage, a combination of $\omega$ and $\varphi$ scans was used, with a step size of $1^{\circ}$ and an exposure time per frame of $3-5$ s. Intensity data were then integrated and corrected for Lorentz polarization effects, using the computer program CrysAlis [20]. An empirical absorption correction was applied using CrysAlis [20]. The structure was refined using SHELX-97 [21]. Scattering curves for neutral chemical species were used at all sites. No peaks larger than $\pm 0.9 \mathrm{e}^{-} / \AA^{3}$ were present in the final difference- the Fourier maps of the electron density and residual maxima and minima were equally balanced. The final agreement index $\left(R_{1}\right)$ was 0.016-0.018/0.017-0.019 (obs/all) for 97 refined parameters. 


\section{Results}

\subsection{Gemmological Properties and 3D Visualisation of the Tourmaline Inclusions}

From a gemmological point of view, the four faceted tourmalines are all transparent, with a colour ranging from green to brown (Figure 2). The specific gravity results from 3.09 to $3.13 \mathrm{~g} / \mathrm{cm}^{3}$, which is typical of elbaitic tourmaline [22]. The optic character is uniaxial negative, and the refractive indices are $n_{\omega}=1.640$ and $n_{\varepsilon}=1.620$ with a birefringence of 0.020 , which is in agreement with the values of elbaite [22]. The samples show a strong dichroism, in the green and brown hues, and they are all inert to ultraviolet radiation. Microscopic observations revealed that the samples contain a few inclusions, typically fluid inclusions, often with a fringed aspect, also called "thrichites" in gemology [23].

More details were provided by the 3D visualisation, which made it possible to display internal features and to quantify the porosity, cracks and voids of fragments from the prismatic portion of samples 7 and 8 .

The volume rendering of the tourmaline sample is reported in Figure $4 \mathrm{a}$, whereas the cross sectional slices, displaying the general appearance of the microstructural features in the grains, are highlighted in Figure 4b,c. In particular, the reconstructed 3D images show that tourmalines are characterised by pores and cracks, the detail of whose volume rendering is displayed in Figure $4 \mathrm{~d}$.
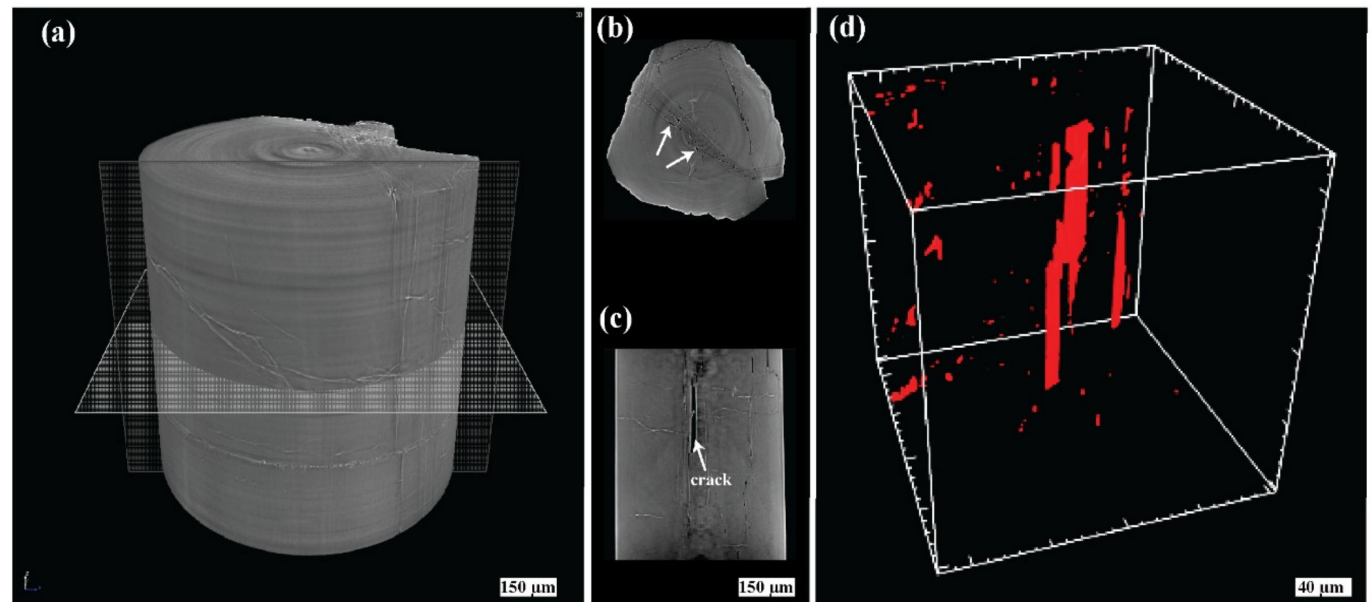

Figure 4. Synchrotron X-ray computed micro-tomography of tourmaline 7: (a) volume rendering of the sample; (b) reconstructed axial slice; (c) the cross section of the stack of reconstructed slices; (d) volume rendering of pores and cracks, a detail.

The total measured porosity accounts for about 0.9 vol. $\%$ and is given as the sum of the voids/pores (0.1 vol.\%) and cracks (0.8 vol.\%), respectively. Please note that the distinction between voids and cracks, which are characterised by the same grey scale values, was performed by considering some morphological features as described in [24]. In particular, the voids appear as spherical bubbles with a small surface/volume ratio (normalized to a sphere) and a low aspect ratio as well. On the contrary, cracks show a medium to medium-high surface/volume ratio, together with high values of aspect ratio.

The rendering pointed out that the voids appear spherical in shape, with an equivalent diameter ranging from 10 to $15 \mu \mathrm{m}$. Morphometric data showed that they are homogeneously distributed within the entire volume and are mostly isolated without any preferred orientation.

Tourmaline crystals show a predominance of crack spacing from the centre to the outer part of the crystals, with a non-homogenous spatial distribution and a heterogeneous size ranging from 20 to $200 \mu \mathrm{m}$. The fractures are mostly flat with a regular surface and appear oriented preferentially parallel to the $z$-axis. Finally, the results of the skeleton analysis pointed out that the crack connectivity is null, because they lack a connected network. 


\subsection{Chemical Composition}

Electron microprobe analyses (EMPA) and backscattered electron (BKSE) images were performed to chemically characterize 10 of the selected samples. Analytical profiles from core to rim or from rim to rim were carried out with step width depending on the crystal size. LA-ICP-MS analyses were performed on the same samples in the areas previously analysed by EMPA-WDS.

The values of $\mathrm{Li}_{2} \mathrm{O}$ measured by LA-ICP-MS indicated that tourmalines contain an average of $1.8 \mathrm{wt} \%$ of $\mathrm{Li}_{2} \mathrm{O}$ (ranging from 0.7 to $2.7 \mathrm{wt} \%$ ), and the result is comparable to that calculated by stoichiometry from the results of the electron microprobe analyses. The studied tourmaline samples exhibit extremely convoluted chemical zoning and are very similar in composition, all classifiable as Li-bearing tourmalines: elbaite $\left(\mathrm{Na}\left(\mathrm{Li}_{1.5}, \mathrm{Al}_{1.5}\right) \mathrm{Al}_{6}\left(\mathrm{BO}_{3}\right)_{3} \mathrm{Si}_{6} \mathrm{O}_{18}(\mathrm{OH})_{4}\right)$, rossmanite $\left(\gamma\left(\mathrm{LiAl}_{2}\right) \mathrm{Al}_{6}\left(\mathrm{BO}_{3}\right)_{3} \mathrm{Si}_{6} \mathrm{O}_{18}(\mathrm{OH})_{4}\right)$, fluor-elbaite $\left(\mathrm{Na}\left(\mathrm{Li}_{1.5}, \mathrm{Al}_{1.5}\right) \mathrm{Al}_{6}\left(\mathrm{BO}_{3}\right)_{3} \mathrm{Si}_{6} \mathrm{O}_{18}(\mathrm{OH})_{3} \mathrm{~F}\right)$ and fluor-liddicoatite $\left(\mathrm{Ca}\left(\mathrm{LiAl}_{2}\right) \mathrm{Al}_{6}\left(\mathrm{BO}_{3}\right)_{3} \mathrm{Si}_{6} \mathrm{O}_{18}(\mathrm{OH})_{3} \mathrm{~F}\right)$. Minor foitite $\left.\left(\gamma \mathrm{Fe}^{2+}{ }_{2} \mathrm{Al}\right) \mathrm{Al}_{6}\left(\mathrm{BO}_{3}\right)_{3} \mathrm{Si}_{6} \mathrm{O}_{18}(\mathrm{OH})_{4}\right)$, a rare tourmaline iron rich in $Y$-site and with more than $50 \%$ vacant $X$-sites [25], was also found.

The complete set of chemical analyses is graphically plotted in the ternary diagram of $X$-site composition (Figure 5).

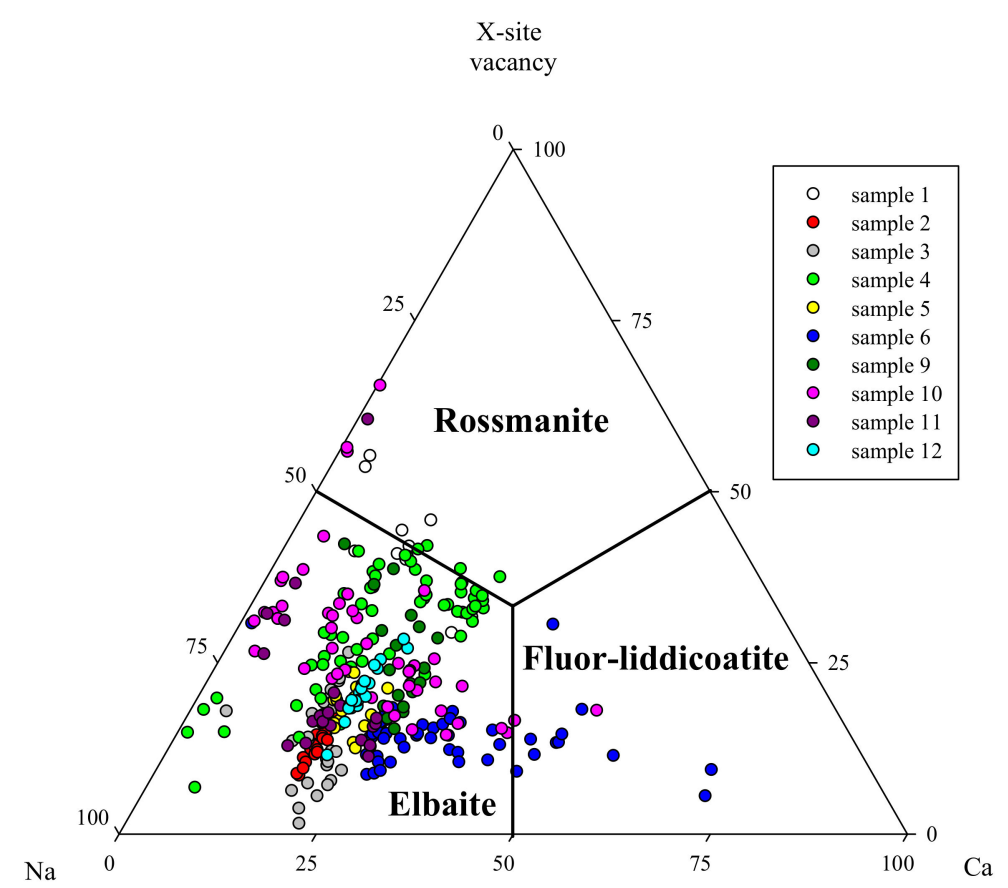

Figure 5. Ternary diagram of $\mathrm{X}$-site composition of all analysed tourmalines.

Most analyses fall in the elbaite/fluor-elbaite field, although a few correspond to rossmanite (samples 1, 4, 10,11) and fluor-liddicoatite (samples 6, 10). Foitite was found at the analogous pole of the crystals of tourmalines 10,11 and as fibrous overgrowth on sample $9 \mathrm{~b}$. The foitite analyses are not reported in the diagram where they would fall in the rossmanite field due to their $>50 \%$ vacant in $X$-site [25].

Selected electron microprobe analyses are reported in Table 2. 
Table 2. Representative electron microprobe analyses of the studied tourmalines.

\begin{tabular}{|c|c|c|c|c|c|c|c|c|c|c|c|c|c|c|c|}
\hline Sample & & & 1 & & 2 & $3 a$ & $3 \mathrm{~b}$ & & & & 4 & & & & 5 \\
\hline & $\begin{array}{c}\text { Pink } \\
\text { Elbaite } \\
\end{array}$ & $\begin{array}{c}\text { Pink } \\
\text { Rossmanite }\end{array}$ & Pink Elbaite & Pink Rossmanite & $\begin{array}{c}\text { Light Blue } \\
\text { Elbaite }\end{array}$ & Colourless Elbaite & $\begin{array}{l}\text { Brown } \\
\text { Elbaite }\end{array}$ & $\begin{array}{c}\text { Rim-Green } \\
\text { Elbaite }\end{array}$ & $\begin{array}{c}\text { Core-Pink } \\
\text { Elbaite }\end{array}$ & $\begin{array}{c}\text { Core-Pink } \\
\text { Elbaite }\end{array}$ & $\begin{array}{c}\text { Core-Pink } \\
\text { Elbaite }\end{array}$ & $\begin{array}{c}\text { Inter-Pink } \\
\text { Elbaite }\end{array}$ & $\begin{array}{c}\text { Rim-Green } \\
\text { Elbaite }\end{array}$ & Elbaite & $\begin{array}{l}\text { Light Green } \\
\text { Elbaite }\end{array}$ \\
\hline & & & & & $\begin{array}{l}\text { Average } \\
\text { (20 pts) }\end{array}$ & Average (10 pts) & $\begin{array}{c}\text { Average } \\
(15 \mathrm{pts})\end{array}$ & & & & & & & & $\begin{array}{l}\text { Average } \\
\text { (20 pts) }\end{array}$ \\
\hline Point Number ${ }^{\circ}$ & 4 & 6 & 8 & 9 & & & & 16 & 10 & 24 & 23 & 56 & 54 & 33 & \\
\hline $\mathrm{SiO}_{2}(\mathrm{wt} \%)$ & 36.34 & 36.63 & 36.28 & 37.06 & 36.2 & 36.17 & 36.54 & 36.99 & 36.79 & 36.67 & 36.59 & 37.04 & 37.28 & 36.71 & 36.29 \\
\hline $\mathrm{TiO}_{2}$ & bdl & bdl & 0.06 & bdl & 0.03 & 0.03 & 0.07 & 0.04 & bdl & bdl & 0.04 & bdl & 0.02 & 0.24 & 0.03 \\
\hline $\mathrm{B}_{2} \mathrm{O}_{3}{ }^{*}$ & 11.06 & 11.18 & 11.02 & 11.27 & 10.78 & 10.87 & 10.77 & 10.98 & 10.94 & 10.93 & 10.91 & 11.04 & 11.05 & 10.64 & 10.9 \\
\hline $\mathrm{Al}_{2} \mathrm{O}_{3}$ & 43.3 & 44.73 & 42.95 & 44.91 & 38.83 & 40.28 & 37.86 & 40.32 & 41.41 & 41.36 & 41.34 & 42.14 & 40.72 & 36.01 & 40.16 \\
\hline & 0.02 & bdl & bdl & bdl & 0.01 & bdl & 0.02 & 0.02 & 0.02 & bdl & bdl & 0.01 & bdl & 0.04 & 0.01 \\
\hline $\mathrm{Cr}_{2} \mathrm{O}_{3}$ & bdl & bdl & bdl & bdl & 0.02 & 0.01 & bdl & bdl & bdl & 0.03 & 0.01 & 0.03 & bdl & 0.01 & bdl \\
\hline $\mathrm{FeO}$ & 0.02 & bdl & bdl & 0.03 & 1.00 & 0.73 & 1.43 & 0.83 & bdl & bdl & 0.01 & 0.01 & 0.52 & 4.75 & 0.77 \\
\hline $\mathrm{MgO}$ & 0.03 & bdl & bdl & 0.01 & 0.02 & 0.02 & 0.01 & 0.01 & bdl & 0.01 & 0.01 & 0.01 & bdl & bdl & 0.01 \\
\hline MnO & 0.52 & 0.21 & 0.55 & 0.27 & 3.79 & 2.79 & 3.99 & 2.82 & 0.16 & 0.16 & 0.13 & 0.12 & 2.02 & 2.45 & 2.93 \\
\hline $\mathrm{ZnO}$ & 0.09 & bdl & bdl & bdl & 0.05 & 0.03 & 0.08 & bdl & 0.14 & 0.08 & 0.05 & 0.03 & 0.10 & 0.50 & 0.05 \\
\hline $\mathrm{CaO}$ & 0.95 & 0.28 & 0.98 & 0.27 & 1.08 & 0.98 & 1.23 & 0.78 & 1.52 & 1.65 & 1.57 & 1.23 & 1.27 & 0.36 & 1.18 \\
\hline $\mathrm{Li}_{2} \mathrm{O}^{*}$ & 1.87 & 1.74 & 1.91 & 1.75 & 1.73 & 1.77 & 1.76 & 1.76 & 2.12 & 2.16 & 2.15 & 2.09 & 1.95 & 1.5 & 1.80 \\
\hline $\mathrm{Na}_{2} \mathrm{O}$ & 1.38 & 1.39 & 1.42 & 1.35 & 2.19 & 2.05 & 2.25 & 1.98 & 1.26 & 1.29 & 1.31 & 1.39 & 1.72 & 2.73 & 1.98 \\
\hline $\mathrm{K}_{2} \mathrm{O}$ & 0.01 & bdl & 0.01 & 0.01 & 0.02 & 0.02 & 0.01 & 0.01 & 0.01 & 0.01 & 0.02 & bdl & bdl & 0.03 & 0.01 \\
\hline $\mathrm{H}_{2} \mathrm{O}^{*}$ & 3.53 & 3.83 & 3.53 & 3.78 & 3.12 & 3.27 & 3.13 & 3.24 & 3.22 & 3.24 & 3.17 & 3.34 & 3.26 & 3.00 & 3.24 \\
\hline $\mathrm{F}$ & 0.61 & 0.07 & 0.57 & 0.24 & 1.27 & 1.00 & 1.25 & 1.16 & 1.17 & 1.11 & 1.25 & 0.98 & 1.16 & 1.41 & 1.10 \\
\hline Total & 99.73 & 100.07 & 99.27 & 100.94 & 100.13 & 100.02 & 100.48 & 100.95 & 98.77 & 98.7 & 98.57 & 99.45 & 101.08 & 100.39 & 100.47 \\
\hline $\mathrm{O}=\mathrm{F}$ & 0.26 & 0.03 & 0.24 & 0.1 & 0.53 & 0.42 & 0.53 & 0.49 & 0.49 & 0.47 & 0.53 & 0.41 & 0.49 & 0.59 & 0.46 \\
\hline Total * & 99.47 & 100.04 & 99.03 & 100.84 & 99.6 & 99.6 & 99.96 & 100.46 & 98.27 & 98.23 & 98.04 & 99.04 & 100.59 & 99.79 & 100 \\
\hline \multicolumn{16}{|c|}{ Structural formula based on 31 anions $(\mathrm{O}, \mathrm{OH}, \mathrm{F})$} \\
\hline $\mathrm{Si}$ & 5.71 & 5.692 & 5.723 & 5.714 & 5.835 & 5.783 & 5.905 & 5.856 & 5.846 & 5.831 & 5.829 & 5.831 & 5.864 & 5.996 & 5.788 \\
\hline $\mathrm{Al}$ & 0.29 & 0.308 & 0.277 & 0.286 & 0.165 & 0.217 & 0.097 & 0.144 & 0.154 & 0.169 & 0.171 & 0.169 & 0.136 & 0.004 & 0.212 \\
\hline$T$ sum & 6.000 & 6.000 & 6.000 & 6.000 & 6.000 & 6.000 & 6.000 & 6.000 & 6.000 & 6.000 & 6.000 & 6.000 & 6.000 & 6.000 & 6.000 \\
\hline B & 3.000 & 3.000 & 3.000 & 3.000 & 3.000 & 3.000 & 3.000 & 3.000 & 3.000 & 3.000 & 3.000 & 3.000 & 3.000 & 3.000 & 3.000 \\
\hline $\mathrm{Al}(\mathrm{Z})$ & 6.000 & 6.000 & 6.000 & 6.000 & 6.000 & 6.000 & 6.000 & 6.000 & 6.000 & 6.000 & 6.000 & 6.000 & 6.000 & 6.000 & 6.000 \\
\hline $\begin{array}{l}\mathrm{Cr} \\
\mathrm{Al}\end{array}$ & 1.727 & 1.884 & 1.709 & 1.875 & 1.211 & 1.373 & 1.081 & 1.379 & 1.601 & 1.583 & 1.59 & 1.65 & 1.413 & 0.928 & 1.336 \\
\hline $\mathrm{Ti}$ & - & - & 0.007 & 0.001 & 0.004 & 0.004 & 0.012 & 0.005 & - & - & 0.004 & - & 0.002 & 0.03 & 0.004 \\
\hline $\mathrm{V}$ & 0.003 & - & - & - & 0.001 & 0.001 & 0.002 & 0.002 & 0.003 & - & & 0.001 & - & 0.006 & 0.001 \\
\hline $\mathrm{Cr}$ & - & - & - & - & 0.002 & 0.001 & 0.001 & - & bdl & 0.004 & 0.002 & 0.003 & - & 0.001 & - \\
\hline $\mathrm{Mg}$ & 0.007 & 0.001 & - & 0.002 & 0.004 & 0.004 & 0.003 & 0.003 & bdl & 0.003 & 0.003 & 0.002 & - & - & 0.003 \\
\hline $\mathrm{Mn}$ & 0.069 & 0.028 & 0.073 & 0.035 & 0.518 & 0.377 & 0.527 & 0.378 & 0.022 & 0.021 & 0.017 & 0.016 & 0.269 & 0.339 & 0.396 \\
\hline $\mathrm{Fe}^{2+}$ & 0.003 & - & - & 0.003 & 0.134 & 0.097 & 0.231 & 0.11 & 0.001 & & 0.001 & 0.001 & 0.069 & 0.649 & 0.102 \\
\hline $\mathrm{Zn}$ & 0.01 & - & - & & 0.006 & 0.004 & 0.013 & & 0.016 & 0.009 & 0.006 & 0.003 & 0.012 & 0.061 & 0.006 \\
\hline $\mathrm{Li}^{*}$ & 1.181 & 1.087 & 1.212 & 1.084 & 1.12 & 1.139 & 1.129 & 1.122 & 1.358 & 1.38 & 1.375 & 1.325 & 1.235 & 0.987 & 1.151 \\
\hline$Y$ sum & 3.000 & 3.000 & 3.000 & 3.000 & 3.000 & 3.000 & 3.000 & 3.000 & 3.000 & 3.000 & 3.000 & 3.000 & 3.000 & 3.000 & 3.000 \\
\hline $\mathrm{Ca}$ & 0.159 & 0.046 & 0.165 & 0.044 & 0.187 & 0.168 & 0.201 & 0.132 & 0.259 & 0.281 & 0.268 & 0.207 & 0.214 & 0.064 & 0.202 \\
\hline $\mathrm{Na}$ & 0.42 & 0.419 & 0.434 & 0.404 & 0.684 & 0.635 & 0.707 & 0.608 & 0.388 & 0.396 & 0.406 & 0.424 & 0.525 & 0.865 & 0.613 \\
\hline $\mathrm{K}$ & 0.002 & & 0.002 & 0.003 & 0.004 & 0.003 & 0.003 & 0.003 & 0.002 & 0.002 & 0.004 & - & - & 0.005 & 0.002 \\
\hline Vacancy & 0.418 & 0.535 & 0.399 & 0.55 & 0.125 & 0.194 & 0.089 & 0.257 & 0.351 & 0.32 & 0.322 & 0.368 & 0.261 & 0.066 & 0.183 \\
\hline$X$ sum & 1.000 & 1.000 & 1.000 & 1.000 & 1.000 & 1.000 & 1.000 & 1.000 & 1.000 & 1.000 & 1.000 & 1.000 & 1.000 & 1.000 & 1.000 \\
\hline $\mathrm{OH}$ & 3.695 & 3.967 & 3.715 & 3.884 & 3.353 & 3.492 & 3.363 & 3.419 & 3.412 & 3.442 & 3.37 & 3.512 & 3.423 & 3.272 & 3.445 \\
\hline F & 0.305 & 0.033 & 0.285 & 0.116 & 0.647 & 0.508 & 0.637 & 0.581 & 0.588 & 0.558 & 0.63 & 0.488 & 0.577 & 0.728 & 0.555 \\
\hline
\end{tabular}


Table 2. Cont

\begin{tabular}{|c|c|c|c|c|c|c|c|c|c|c|c|c|c|c|c|c|}
\hline \multirow[t]{3}{*}{ Sample } & \multirow[b]{3}{*}{$\begin{array}{l}\text { Light } \\
\text { Yellow } \\
\text { Elbaite }\end{array}$} & \multirow[b]{3}{*}{$\begin{array}{l}\text { Light } \\
\text { Yellow } \\
\text { Elbaite }\end{array}$} & \multirow[t]{2}{*}{6} & \multirow[b]{3}{*}{$\begin{array}{c}\text { Colourless } \\
\text { Flour-Liddicoatite }\end{array}$} & \multirow{3}{*}{\begin{tabular}{|c|} 
9a \\
$\begin{array}{c}\text { Pink } \\
\text { Elbaite }\end{array}$ \\
\end{tabular}} & \multicolumn{2}{|c|}{$9 \mathrm{~b}$} & \multirow{2}{*}{\multicolumn{3}{|c|}{ Analogous Green }} & \multirow{2}{*}{\multicolumn{3}{|c|}{$\begin{array}{c}10 \\
\text { Antilogous Dark Green }\end{array}$}} & \multirow[b]{3}{*}{ Rossmanite } & \multirow{3}{*}{$\begin{array}{c}11 \\
\begin{array}{c}\text { Green } \\
\text { Elbaite }\end{array}\end{array}$} & \multirow[t]{2}{*}{12} \\
\hline & & & & & & & & & & & & & & & & \\
\hline & & & $\begin{array}{l}\text { Light Yellow } \\
\text { Fluor-Liddicoatite }\end{array}$ & & & $\begin{array}{l}\text { Green } \\
\text { Elbaite }\end{array}$ & Foitite & Elbaite & Elbaite & Elbaite & Fluor-Liddicoatite & Elbaite & Elbaite & & & Blue Elbaite \\
\hline & & & & & $\begin{array}{c}\text { Average } \\
(9 \mathrm{pts})\end{array}$ & $\begin{array}{c}\text { Average } \\
(10 \mathrm{pts})\end{array}$ & & & & & & & & & $\begin{array}{c}\text { Average } \\
(21 \mathrm{pts})\end{array}$ & $\begin{array}{l}\text { Average } \\
(20 \text { pts) }\end{array}$ \\
\hline Point Number ${ }^{\circ}$ & & & & & & & & 4 & 10 & 12 & 36 & 42 & 43 & 45 & & \\
\hline $\mathrm{SiO}_{2}(\mathrm{wt} \%)$ & 35.70 & 36.00 & 36.71 & 37.50 & 37.12 & 37.32 & 35.82 & 37.38 & 38.13 & 37.51 & 37.60 & 37.82 & 37.13 & 37.06 & 36.95 & 37.10 \\
\hline $\mathrm{TiO}_{2}$ & 0.14 & 0.17 & 0.09 & 0.09 & 0.01 & 0.17 & bdl & bdl & bdl & 0.18 & 0.03 & 0.05 & 0.20 & 0.07 & 0.18 & 0.17 \\
\hline $\mathrm{B}_{2} \mathrm{O}_{3}{ }^{*}$ & 10.84 & 10.83 & 10.94 & 10.99 & 11.07 & 11.00 & 10.39 & 10.92 & 11.09 & 11.02 & 10.99 & 11.04 & 10.98 & 10.76 & 10.96 & 10.93 \\
\hline $\mathrm{Al}_{2} \mathrm{O}_{3}$ & 40.53 & 39.68 & 39.90 & 39.43 & 41.59 & 39.78 & 33.55 & 39.33 & 39.60 & 39.74 & 39.03 & 40.09 & 39.60 & 37.57 & 39.86 & 39.30 \\
\hline $\mathrm{V}_{2} \mathrm{O}_{3}$ & 0.01 & 0.04 & 0.01 & bdl & 0.01 & 0.02 & bdl & 0.03 & bdl & 0.10 & 0.02 & bdl & 0.04 & bdl & 0.01 & bdl \\
\hline $\mathrm{Cr}_{2} \mathrm{O}_{3}$ & bdl & bdl & bdl & 0.01 & 0.01 & 0.01 & 0.01 & 0.01 & bdl & bdl & 0.02 & bdl & 0.05 & bdl & 0.01 & 0.01 \\
\hline $\mathrm{FeO}$ & 0.34 & 0.54 & 0.22 & 0.16 & 0.08 & 0.71 & 11.52 & 1.68 & 0.44 & 0.85 & 0.34 & 0.88 & 1.25 & 3.40 & 1.18 & 0.66 \\
\hline $\mathrm{MgO}$ & 0.01 & 0.01 & 0.01 & bdl & 0.01 & 0.01 & 2.57 & 0.03 & 0.01 & 0.01 & 0.02 & 0.05 & 0.04 & 0.02 & 0.03 & 0.03 \\
\hline $\mathrm{MnO}$ & 2.38 & 2.51 & 1.29 & 0.99 & 1.60 & 2.05 & 0.17 & 3.68 & 1.82 & 1.82 & 1.45 & 2.89 & 1.87 & 5.67 & 2.31 & 3.10 \\
\hline $\mathrm{ZnO}$ & bdl & bdl & 0.08 & bdl & 0.03 & 0.06 & 0.10 & 0.12 & 0.12 & 0.02 & 0.08 & 0.09 & 0.19 & 0.18 & 0.02 & 0.03 \\
\hline $\mathrm{CaO}$ & 1.49 & 1.58 & 2.67 & 2.94 & 1.16 & 1.53 & 0.02 & 0.13 & 1.96 & 1.62 & 3.05 & 0.27 & 1.47 & 0.07 & 1.00 & 1.21 \\
\hline $\mathrm{Li}_{2} \mathrm{O}^{*}$ & 1.90 & 1.97 & 2.31 & 2.44 & 1.96 & 2.05 & 0.14 & 1.45 & 2.20 & 2.05 & 2.38 & 1.71 & 1.98 & 0.91 & 1.87 & 1.88 \\
\hline $\mathrm{Na}_{2} \mathrm{O}$ & 1.81 & 1.97 & 1.32 & $\begin{array}{l}1.05 \\
b y 1\end{array}$ & 1.59 & 1.77 & 1.48 & 1.97 & $\begin{array}{l}1.50 \\
h y\end{array}$ & 1.54 & 0.99 & 2.10 & 1.84 & 1.37 & 2.08 & 1.88 \\
\hline $\begin{array}{l}\mathrm{K}_{2} \mathrm{O} \\
\mathrm{H} 2 \mathrm{O}\end{array}$ & bdl & 0.02 & 0.01 & bdl & 0.01 & 0.01 & 0.01 & 0.01 & bdl & 0.01 & 0.01 & bdl & 0.01 & 0.02 & 0.02 & 0.02 \\
\hline$\frac{\mathrm{H}_{2} \mathrm{O}^{*}}{\mathrm{~F}}$ & $\begin{array}{l}3.29 \\
0.95\end{array}$ & $\begin{array}{l}3.17 \\
119\end{array}$ & $\begin{array}{l}3.23 \\
116\end{array}$ & $\begin{array}{l}3.21 \\
123\end{array}$ & $\begin{array}{l}3.39 \\
0.91\end{array}$ & $\begin{array}{l}3.25 \\
114\end{array}$ & $\begin{array}{l}3.58 \\
\text { bdl }\end{array}$ & 3.77 & $\begin{array}{l}3.45 \\
079\end{array}$ & 3.22 & $\begin{array}{l}3.18 \\
179\end{array}$ & $\begin{array}{l}3.81 \\
\text { bdd }\end{array}$ & $\begin{array}{l}3.28 \\
108\end{array}$ & $\begin{array}{l}3.71 \\
\text { bd1 }\end{array}$ & $\begin{array}{r}3.33 \\
0.95\end{array}$ & $\begin{array}{l}3.22 \\
117\end{array}$ \\
\hline Total & $\begin{array}{l}0.95 \\
99.39\end{array}$ & $\begin{array}{l}1.19 \\
99.67\end{array}$ & $\begin{array}{l}1.16 \\
99.94\end{array}$ & $\begin{array}{c}1.23 \\
\text {. }\end{array}$ & $\begin{array}{c}0.91 \\
10054\end{array}$ & $\begin{array}{c}1.14 \\
10088\end{array}$ & $\begin{array}{l}\text { bdl } \\
99937\end{array}$ & $\begin{array}{l}\text { bdl } \\
100.5\end{array}$ & $\begin{array}{c}0.79 \\
10911\end{array}$ & $\begin{array}{c}1.23 \\
0\end{array}$ & $\begin{array}{l}1.29 \\
\end{array}$ & bdl & 1.08 & bdl & $\begin{array}{c}0.95 \\
1\end{array}$ & $\begin{array}{l}1.17 \\
1007\end{array}$ \\
\hline $\mathrm{O}=\mathrm{F}$ & 0.40 & 0.50 & $\begin{array}{c}09.94 \\
0.49\end{array}$ & $\begin{array}{c}1000.03 \\
0.52\end{array}$ & $\begin{array}{c}100.54 \\
0.38\end{array}$ & 0.48 & $\begin{array}{c}99.37 \\
\text { bdl }\end{array}$ & $\begin{array}{c}\begin{array}{c}100.52 \\
\text { bdl }\end{array} \\
\text { bd }\end{array}$ & $\begin{array}{c}101.11 \\
0.33\end{array}$ & $\begin{array}{c}100.90 \\
0.52\end{array}$ & $\begin{array}{c}100.49 \\
0.54\end{array}$ & $\begin{array}{c}100.81 \\
\text { bdl }\end{array}$ & $\begin{array}{l}101.00 \\
0.45\end{array}$ & $\begin{array}{c}100.82 \\
\text { bdl }\end{array}$ & $\begin{array}{c}100.77 \\
0.40\end{array}$ & $\begin{array}{c}100.70 \\
0.49\end{array}$ \\
\hline Total * & 98.99 & 99.17 & 99.46 & 99.52 & 100.16 & 100.40 & 99.37 & 100.52 & 100.78 & 100.38 & 99.95 & 100.81 & 100.55 & 100.82 & 100.37 & 100.21 \\
\hline \multicolumn{17}{|c|}{ Structural formula based on 31 anions $(\mathrm{O}, \mathrm{OH}, \mathrm{F})$} \\
\hline $\mathrm{Si}$ & 5.726 & 5.779 & 5.831 & 5.931 & 5.830 & 5.894 & 5.994 & 5.948 & 5.978 & 5.918 & 5.946 & 5.952 & 5.875 & 5.985 & 5.858 & 5.898 \\
\hline $\mathrm{Al}$ & 0.274 & 0.221 & 0.169 & 0.069 & 0.170 & 0.106 & 0.006 & 0.052 & 0.022 & 0.082 & 0.054 & 0.048 & 0.125 & 0.015 & 0.142 & 0.102 \\
\hline$T$ sum & 6.000 & 6.000 & 6.000 & 6.000 & 6.000 & 6.000 & 6.000 & 6.000 & 6.000 & 6.000 & 6.000 & 6.000 & 6.000 & 6.000 & 6.000 & 6.000 \\
\hline$B$ & 3.000 & 3.000 & 3.000 & 3.000 & 3.000 & 3.000 & 3.000 & 3.000 & 3.000 & 3.000 & 3.000 & 3.000 & 3.000 & 3.000 & 3.000 & 3.000 \\
\hline $\mathrm{Al}(\mathrm{Z})$ & 6.000 & 6.000 & 6.000 & 6.000 & 6.000 & 6.000 & 6.000 & 6.000 & 6.000 & 6.000 & 6.000 & 6.000 & 6.000 & 6.000 & 6.000 & 6.000 \\
\hline $\mathrm{Cr}$ & 1287 & & & & & & & 1323 & & & & & 1260 & & & \\
\hline $\mathrm{Al}$ & 1.387 & 1.287 & 1.301 & 1.282 & 1.528 & 1.299 & 0.612 & 1.323 & 1.296 & 1.307 & 1.220 & 1.387 & 1.260 & 1.136 & 1.305 & 1.263 \\
\hline $\mathrm{Ti}$ & 0.017 & 0.020 & 0.011 & 0.010 & 0.001 & 0.020 & - & - & - & 0.021 & 0.004 & 0.006 & 0.024 & 0.008 & 0.022 & 0.020 \\
\hline $\mathrm{V}$ & 0.002 & 0.005 & 0.001 & - & 0.001 & 0.002 & - & 0.004 & - & 0.012 & 0.003 & - & 0.005 & - & 0.001 & 0.001 \\
\hline $\mathrm{Cr}$ & 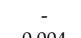 & 0000 & ח 002 & 0.001 & 0.001 & 0.001 & 0.002 & 0.001 & 0000 & 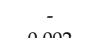 & 0.003 & 0018 & 0.006 & - & 0.001 & 0.001 \\
\hline $\mathrm{Mg}$ & $\begin{array}{l}0.004 \\
0323\end{array}$ & $\begin{array}{l}0.003 \\
0.303\end{array}$ & $\begin{array}{l}0.003 \\
0173\end{array}$ & 0.001 & 0.001 & 0.003 & 0.641 & $\begin{array}{l}0.008 \\
\end{array}$ & 0.002 & $\begin{array}{l}0.002 \\
0.43\end{array}$ & $\begin{array}{l}0.005 \\
0.194\end{array}$ & $\begin{array}{l}0.012 \\
0389\end{array}$ & $\begin{array}{l}0.009 \\
0.259\end{array}$ & 0.006 & 0.008 & 0.007 \\
\hline $\mathrm{Mn}$ & 0.323 & 0.341 & 0.173 & 0.133 & 0.213 & 0.275 & 0.024 & 0.496 & 0.242 & 0.243 & 0.194 & 0.385 & 0.251 & 0.776 & 0.310 & 0.417 \\
\hline $\mathrm{Fe}^{2+}$ & 0.046 & 0.072 & 0.029 & 0.021 & 0.010 & 0.093 & 1.612 & 0.224 & 0.058 & 0.112 & 0.045 & 0.116 & 0.165 & 0.459 & 0.157 & 0.087 \\
\hline $\mathrm{Zn}$ & & & 0.010 & & 0.004 & 0.007 & 0.013 & 0.014 & 0.014 & 0.002 & 0.010 & 0.010 & 0.022 & 0.022 & 0.003 & 0.003 \\
\hline $\mathrm{Li}^{*}$ & 1.223 & 1.271 & 1.473 & 1.552 & 1.240 & 1.299 & 0.096 & 0.930 & 1.389 & 1.300 & 1.517 & 1.083 & 1.259 & 0.594 & 1.193 & 1.200 \\
\hline$Y$ sum & 3.000 & 3.000 & 3.000 & 3.000 & 3.000 & 3.000 & 3.000 & 3.000 & 3.000 & $\begin{array}{l}3.000 \\
0.070\end{array}$ & $\begin{array}{l}3.000 \\
\end{array}$ & 3.000 & 3.000 & 3.000 & 3.000 & 3.000 \\
\hline $\mathrm{Ca}$ & 0.256 & 0.272 & 0.454 & 0.498 & 0.195 & 0.259 & 0.003 & 0.022 & 0.329 & 0.274 & 0.517 & 0.046 & 0.249 & 0.012 & 0.171 & 0.207 \\
\hline $\mathrm{Na}$ & 0.563 & 0.613 & 0.407 & 0.321 & 0.485 & 0.542 & 0.480 & 0.608 & 0.456 & 0.471 & 0.303 & 0.641 & 0.564 & 0.429 & 0.640 & 0.581 \\
\hline K & 0.001 & 0.003 & 0.002 & 0.000 & 0.002 & 0.002 & 0.002 & 0.002 & & 0.001 & 0.002 & & 0.003 & 0.003 & 0.003 & 0.004 \\
\hline $\begin{array}{l}\text { Vacancy } \\
X\end{array}$ & 0.181 & 0.112 & 0.137 & 0.180 & 0.317 & 0.197 & 0.515 & 0.368 & 0.214 & 0.254 & 0.179 & 0.313 & 0.184 & 0.555 & 0.187 & 0.209 \\
\hline$X$ sum & 1.000 & 1.000 & 1.000 & 1.000 & 1.000 & 1.000 & 1.000 & 1.000 & 1.000 & 1.000 & 1.000 & 1.000 & 1.000 & 1.000 & 1.000 & 1.000 \\
\hline $\mathrm{OH}_{\mathrm{F}}$ & $\begin{array}{l}3.519 \\
0481\end{array}$ & $\begin{array}{l}3.396 \\
0.604\end{array}$ & $\begin{array}{l}3.417 \\
0.583\end{array}$ & $\begin{array}{l}3.385 \\
0.615\end{array}$ & 3.547 & $\begin{array}{l}3.429 \\
0577\end{array}$ & 4.000 & 4.000 & $\begin{array}{l}3.608 \\
0.399\end{array}$ & $\begin{array}{l}3.386 \\
0.674\end{array}$ & $\begin{array}{l}3.355 \\
0.654\end{array}$ & 4.000 & $\begin{array}{l}3.460 \\
054\end{array}$ & 4.000 & 3.522 & $\begin{array}{l}3.412 \\
0588\end{array}$ \\
\hline & 0.481 & 0.604 & 0.583 & 0.615 & 0.453 & 0.571 & & & 0.392 & 0.614 & 0.645 & & 0.540 & & 0.478 & 0.588 \\
\hline
\end{tabular}

* calculated on the assumed elbaite stoichiometry. $\mathrm{bdl}=$ below the detection limit $\left(0.01 \mathrm{wt} \%\right.$ for all oxides). ${ }^{\circ}$ point number corresponding to the number in the sample images, pts $=$ points. 
Colour changes are generally controlled by a combination of concentration and oxidation states of chromophore elements such as Ti, V, Fe and Mn [26]: in samples 3, 10 and 11, a higher content of iron is detected in the portion typically brown-black. Sample 10 (Figure 6a,b) is the only one including the analogous and antilogous pole and shows compositional variations with changes in Mn, Fe (Figure 6c) and Ca. This last displays the highest values in the central part of the crystal (Figure 6d). Titanium ranges from 0 to 0.04 a.p.f.u., whereas $\mathrm{V}$ and $\mathrm{Cr}$ are very low or below the detection limit. Rossmanite is present both at the analogous and antilogous poles (Figure 6b).
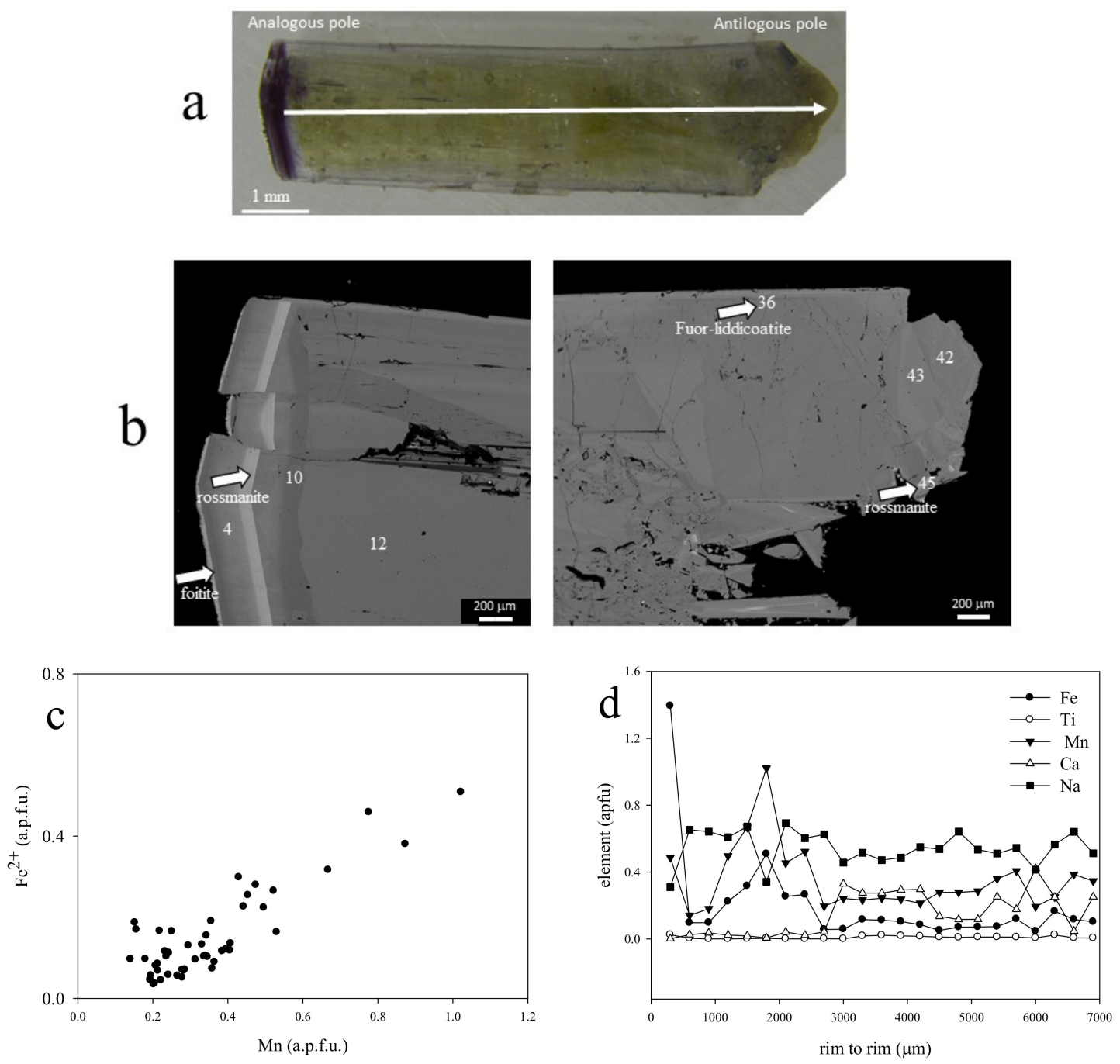

Figure 6. Optical microscope (a), BKSE (b) images, (c) variation of Fe vs. Mn and (d) an example of zoning patterns of different elements of sample 10. The points in BKSE images correspond to the points reported in Table 2.

Sample 3 (Figure 7a), along the $c$-axis, shows a colourless (a) and a brown (b) zone with an iron content of up to 0.106 and up to 0.762 a.p.f.u., respectively. Sample 6 shows an elbaite composition with a decrease of $\mathrm{Mn}$ and $\mathrm{Na}$ together with an increase of $\mathrm{Ca}$ from light yellow to near colourless zones where a fluor-liddicoatite composition is identified (Figure $7 \mathrm{~b}, \mathrm{c}$ ). Ti and Fe are very low, and therefore manganese content seems to be mainly responsible of the colour changes.

Samples 2 and 12 have a homogeneous composition with the higher content of Mn that may induce the deep blue colour (up to 0.548 and 0.443 a.p.f.u., respectively). 

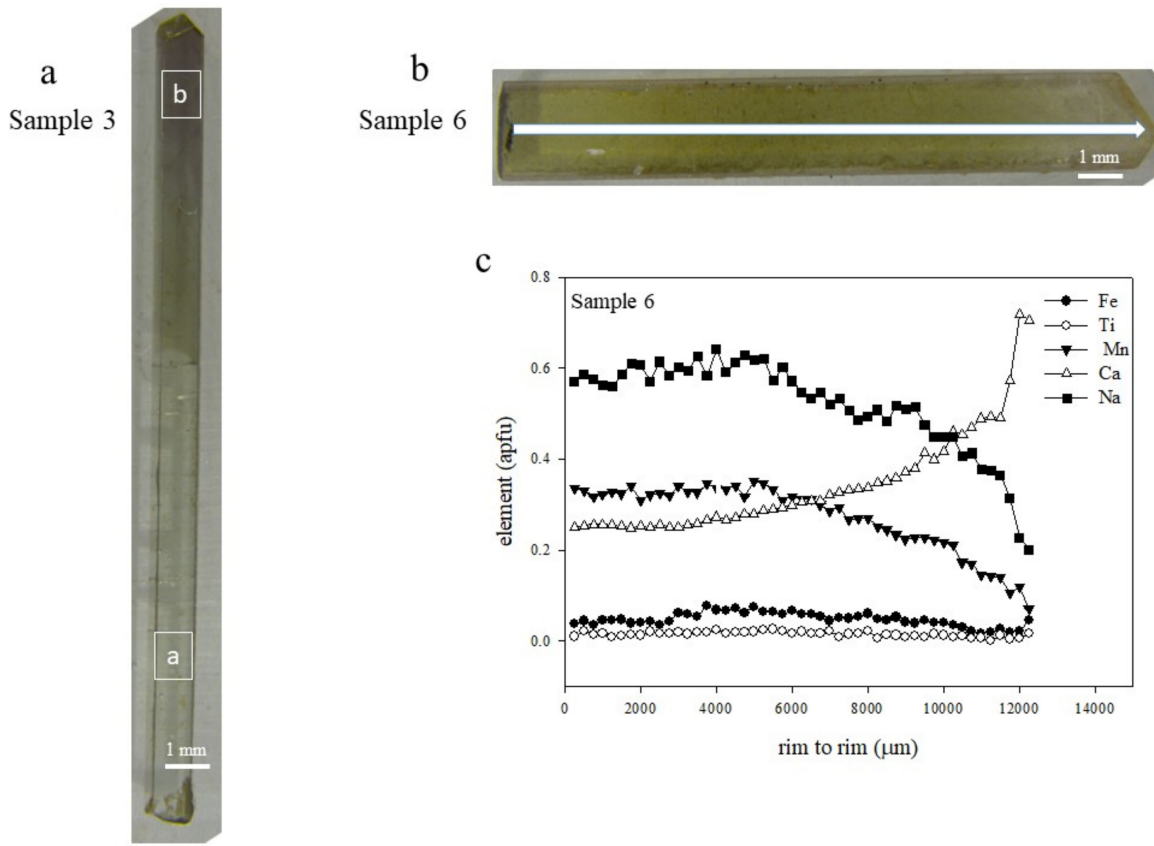

Figure 7. Optical microscope image of sample 3 (a) and sample $6(\mathbf{b})$; zoning patterns of different elements of sample 6 (c).

The pink tourmaline (sample 1, Figure 8a) is nearly devoid of iron and presents the lowest Mn content with two different compositional zones: one with higher $\mathrm{Ca}+\mathrm{Mn}$ (on average, 0.27 a.p.f.u., i.e., pts 4 and 8), the second one with lower $\mathrm{Ca}+\mathrm{Mn}$ (on average, 0.12 a.p.f.u.), $\mathrm{Mn}<0.04$ a.p.f.u. and higher mole fraction of rossmanite (i.e., pts 6 and 9). Lighter-coloured areas included in rossmanite resulted in being muscovite (Figure $8 b$ ).

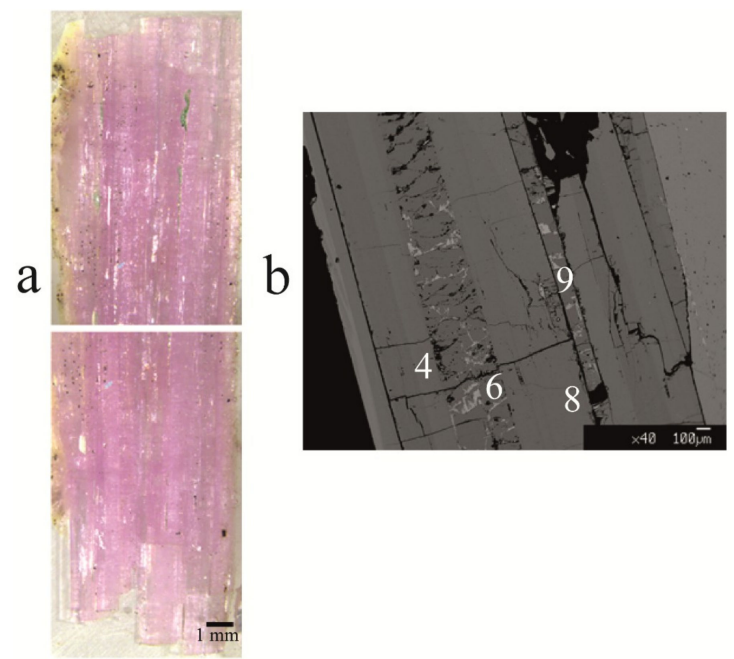

Figure 8. Optical microscope (a) and BKSE (b) images of sample 1. The points in BKSE image correspond to the points reported in Table 2. Points 6 and 9 correspond to rossmannite.

Sample 4 (Figure 9a) occurs in the same geode of sample 3 and has been cut perpendicular to the $c$-axis. The slice reveals a complex growth history characterized by a central part, pink in colour, depleted in $\mathrm{Mn}$ and $\mathrm{Fe}$, but enriched in $\mathrm{Ca}$ with respect to the green rim, with a composition ranging from fluor-liddicoatite-rich elbaite to elbaite. The lighter-coloured zone at the bottom of the sample (Figure 9b, e.g., point 33, analysis 33 in Table 2) presents an enrichment in $\mathrm{Fe}^{2+}$ with respect to $\mathrm{Mn}^{2+}$ (Figure 9c). In Figure 9d, an example of the variation from rim to rim of these elements, including Ti, $\mathrm{Ca}$ and $\mathrm{Na}$, is shown. 

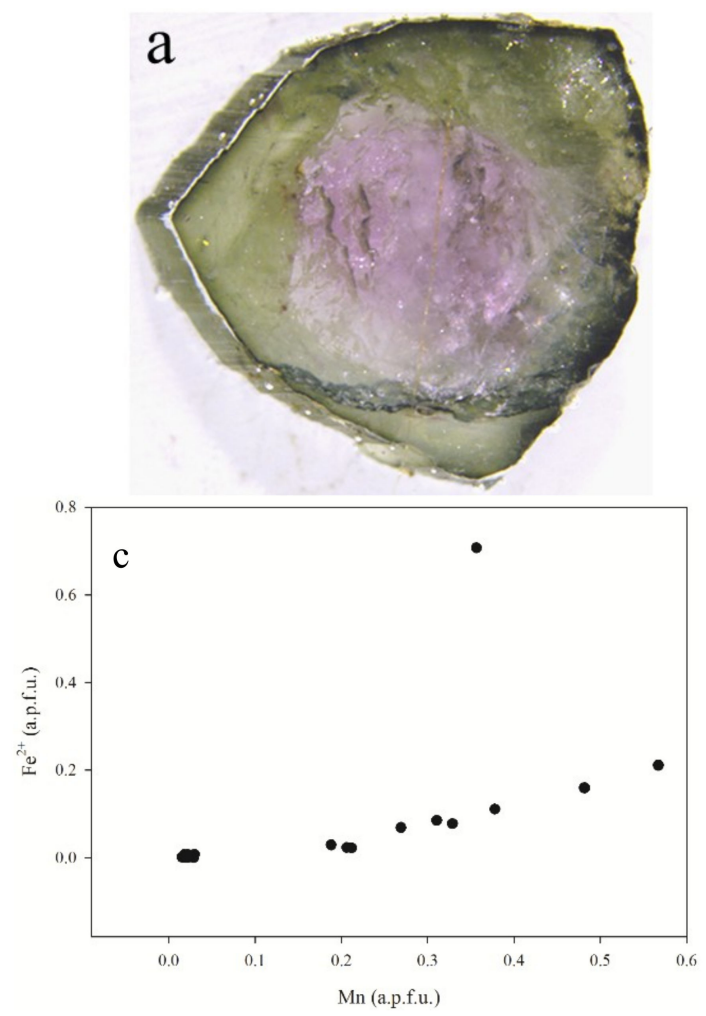
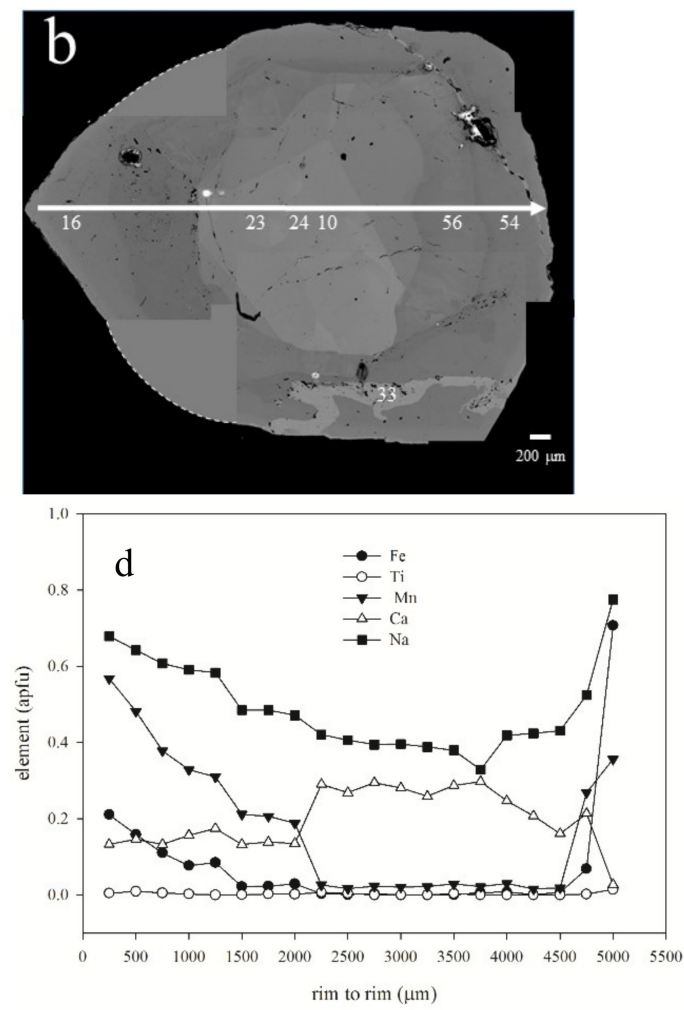

Figure 9. Optical microscope (a) and BKSE (b) images, (c) variation of Fe vs. Mn and (d) an example of zoning patterns of different elements of sample 4. The points in BKSE image correspond to the points reported in Table 2.

Sample 9 (Figure 10a,b) comes from a geode containing black quartz, zircon and uraninite and displays a change from pink (sample 9a) to green colour (sample 9b) that seems to be due to manganese, iron and titanium enrichment. In sample 9b, a thin overgrowth with foitite composition (see Figure 10c and Table 2) is present.
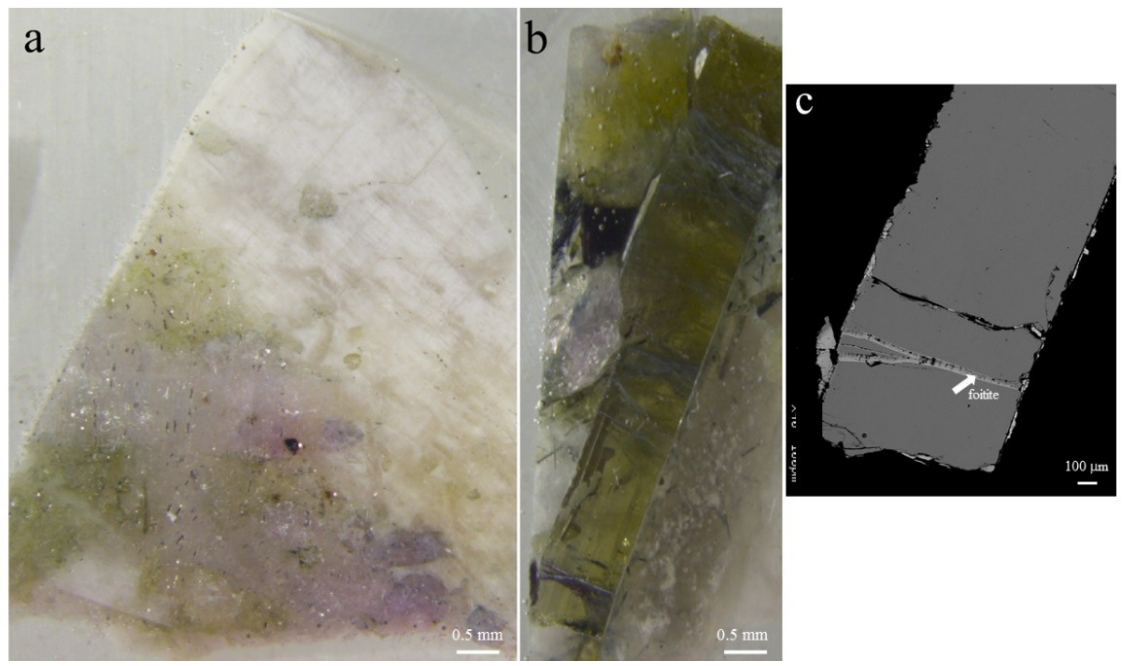

Figure 10. Optical microscope images of sample 9a (a), 9b (b) and BSKE image of sample 9b (c) where the arrow indicates the foitite.

The averaged LA-ICP-MS analyses of selected trace elements: $\mathrm{Be}, \mathrm{Sc}, \mathrm{V}, \mathrm{Cu}, \mathrm{Ga}, \mathrm{Ge}, \mathrm{Sr}, \mathrm{Y}, \mathrm{Ta}, \mathrm{Pb}$, Th, $\mathrm{U}$ and REE of the tourmalines are reported in Table 3. 
Table 3. LA-ICP-MS analyses of trace elements in studied tourmalines (ppm).

\begin{tabular}{|c|c|c|c|c|c|c|c|c|c|c|c|c|}
\hline \multirow[t]{2}{*}{ Sample } & \multicolumn{2}{|c|}{1} & \multicolumn{2}{|c|}{2} & \multicolumn{2}{|c|}{$3 a$} & \multicolumn{2}{|c|}{$3 b$} & \multicolumn{2}{|c|}{4} & \multicolumn{2}{|c|}{5} \\
\hline & $\begin{array}{c}\text { Average } \\
\text { (4 pts) }\end{array}$ & st dev & $\begin{array}{c}\text { Average } \\
\text { (4 pts) }\end{array}$ & st dev & $\begin{array}{c}\text { Average } \\
\text { (4 pts) }\end{array}$ & st dev & $\begin{array}{c}\text { Average } \\
\text { (5 pts) }\end{array}$ & st dev & $\begin{array}{c}\text { Average } \\
\text { (8 pts) }\end{array}$ & st dev & $\begin{array}{c}\text { Average } \\
\text { (5 pts) }\end{array}$ & st dev \\
\hline $\mathrm{Li}$ & 8546.20 & 463.86 & 7985.55 & 182.95 & 7859.06 & 75.06 & 7919.98 & 324.03 & 8899.59 & 1020.41 & 8073.58 & 322.46 \\
\hline Be & 52.19 & 14.18 & 37.62 & 2.83 & 28.92 & 2.96 & 21.29 & 8.82 & 99.38 & 122.07 & 31.05 & 9.50 \\
\hline Sc & 3.03 & 0.55 & 1.83 & 0.28 & 1.82 & 0.35 & 2.79 & 0.45 & 1.63 & 0.52 & 2.12 & 0.16 \\
\hline $\mathrm{V}$ & 0.38 & 0.32 & 1.08 & 0.20 & 0.82 & 0.12 & 13.95 & 7.92 & 8.56 & 5.26 & 3.17 & 4.77 \\
\hline $\mathrm{Cu}$ & 196.20 & 27.01 & 19.23 & 1.05 & 16.10 & 1.41 & 16.04 & 12.69 & 71.89 & 62.62 & 22.16 & 3.19 \\
\hline $\mathrm{Ga}$ & 181.73 & 28.06 & 40.83 & 2.51 & 45.27 & 1.04 & 59.90 & 13.21 & 78.28 & 26.48 & 44.19 & 6.70 \\
\hline $\mathrm{Ge}$ & 9.11 & 2.22 & 20.47 & 1.68 & 19.85 & 1.52 & 15.65 & 2.90 & 15.91 & 2.78 & 21.57 & 2.77 \\
\hline $\mathrm{Sr}$ & 0.73 & 0.23 & 31.66 & 1.23 & 19.88 & 1.72 & 231.22 & 390.52 & 93.98 & 234.91 & 29.42 & 3.66 \\
\hline $\mathrm{Y}$ & 0.25 & 0.17 & 0.46 & 0.05 & 0.35 & 0.03 & 0.24 & 0.15 & 0.22 & 0.08 & 0.39 & 0.20 \\
\hline $\mathrm{Ta}$ & 4.63 & 3.57 & 1.07 & 0.14 & 0.28 & 0.01 & 0.56 & 0.31 & 3.18 & 5.43 & 0.44 & 0.40 \\
\hline $\mathrm{Pb}$ & 11.94 & 4.76 & 43.78 & 2.22 & 35.05 & 1.19 & 2882.66 & 3775.66 & 184.51 & 453.38 & 163.22 & 71.23 \\
\hline Th & 3.58 & 2.95 & 0.79 & 0.06 & 0.55 & 0.06 & 0.16 & 0.14 & 6.98 & 15.66 & 0.52 & 0.32 \\
\hline $\mathrm{U}$ & 0.36 & 0.29 & 0.02 & 0.00 & bdl & & 0.01 & 0.01 & 0.79 & 1.15 & 0.03 & \\
\hline $\mathrm{La}$ & 44.13 & 18.50 & 24.29 & 1.46 & 13.58 & 0.35 & 22.86 & 14.36 & 9.50 & 4.54 & 17.07 & 4.19 \\
\hline $\mathrm{Ce}$ & 46.68 & 19.98 & 49.72 & 3.99 & 30.16 & 0.30 & 48.87 & 26.13 & 15.89 & 8.20 & 37.41 & 11.14 \\
\hline $\operatorname{Pr}$ & 2.35 & 1.08 & 5.13 & 0.38 & 2.87 & 0.10 & 4.94 & 2.44 & 1.44 & 0.85 & 3.60 & 1.08 \\
\hline $\mathrm{Nd}$ & 3.05 & 1.40 & 14.54 & 1.49 & 7.46 & 0.24 & 13.43 & 6.78 & 3.42 & 2.55 & 9.15 & 2.88 \\
\hline $\mathrm{Sm}$ & 0.53 & 0.36 & 3.14 & 0.69 & 1.96 & 0.17 & 2.74 & 1.23 & 1.17 & 0.41 & 2.29 & 0.95 \\
\hline $\mathrm{Eu}$ & bdl & & 0.12 & 0.03 & 0.12 & 0.04 & 0.13 & 0.05 & 0.05 & 0.02 & 0.10 & 0.04 \\
\hline $\mathrm{Gd}$ & 0.27 & 0.02 & 0.93 & 0.19 & 0.64 & 0.13 & 0.68 & 0.40 & 0.42 & 0.16 & 0.72 & 0.38 \\
\hline $\mathrm{Tb}$ & 0.05 & 0.04 & 0.11 & 0.02 & 0.09 & 0.02 & 0.08 & 0.05 & 0.05 & 0.02 & 0.09 & 0.04 \\
\hline Dy & 0.15 & 0.17 & 0.36 & 0.11 & 0.24 & 0.05 & 0.24 & 0.17 & 0.14 & 0.04 & 0.26 & 0.17 \\
\hline Ho & bdl & & bdl & & 0.03 & 0.02 & 0.02 & 0.01 & 0.02 & 0.01 & 0.02 & 0.01 \\
\hline Er & 0.20 & 0.26 & bdl & & 0.05 & 0.001 & bdl & & 0.06 & 0.03 & bdl & \\
\hline $\mathrm{Tm}$ & bdl & & 0.02 & 0.005 & 0.01 & 0.005 & 0.01 & 0.00 & bdl & & bdl & \\
\hline $\mathrm{Yb}$ & 0.10 & 0.10 & bdl & & & & bdl & & 0.17 & 0.15 & bdl & \\
\hline $\mathrm{Lu}$ & bdl & & bdl & & bdl & & bdl & & bdl & & 0.01 & \\
\hline$\Sigma$ REE & 97.50 & & 98.36 & & 57.21 & & 94.00 & & 32.34 & & 70.72 & \\
\hline
\end{tabular}


Table 3. Cont.

\begin{tabular}{|c|c|c|c|c|c|c|c|c|c|c|c|c|}
\hline \multirow[t]{2}{*}{ Sample } & \multicolumn{2}{|c|}{6} & \multicolumn{2}{|c|}{$9 a$} & \multicolumn{2}{|c|}{$9 b$} & \multicolumn{2}{|c|}{10} & \multicolumn{2}{|c|}{11} & \multicolumn{2}{|c|}{12} \\
\hline & $\begin{array}{l}\text { Average } \\
\text { (7 pts) }\end{array}$ & st dev & $\begin{array}{l}\text { Average } \\
(3 \text { pts })\end{array}$ & st dev & $\begin{array}{c}\text { Average } \\
\text { (3 pts) }\end{array}$ & st dev & $\begin{array}{l}\text { Average } \\
(8 \mathrm{pts})\end{array}$ & st dev & $\begin{array}{c}\text { Average } \\
(5 \text { pts })\end{array}$ & st dev & $\begin{array}{c}\text { Average } \\
\text { (4 pts) }\end{array}$ & st dev \\
\hline $\mathrm{Li}$ & 9993.23 & 1095.08 & 8494.71 & 249.68 & 8767.86 & 145.96 & 8444.96 & 1786.74 & 7175.63 & 2373.44 & 8271.11 & 399.03 \\
\hline $\mathrm{Be}$ & 20.20 & 5.78 & 23.17 & 2.81 & 27.37 & 7.68 & 22.63 & 13.36 & 14.62 & 11.00 & 19.95 & 2.56 \\
\hline Sc & 1.96 & 0.40 & 2.96 & 0.25 & 3.09 & 0.24 & 2.26 & 0.46 & 3.47 & 0.84 & 1.65 & 0.47 \\
\hline V & 0.37 & 0.09 & 0.48 & 0.18 & 8.88 & 3.03 & 0.84 & 0.63 & 1.21 & 0.54 & 0.54 & 0.09 \\
\hline $\mathrm{Cu}$ & 21.30 & 9.49 & 148.85 & 58.16 & 31.74 & 3.36 & 24.96 & 16.28 & 17.92 & 12.74 & 30.46 & 6.96 \\
\hline $\mathrm{Ga}$ & 68.20 & 25.78 & 121.62 & 49.09 & 35.29 & 1.08 & 104.43 & 26.62 & 91.33 & 12.88 & 45.58 & 5.77 \\
\hline $\mathrm{Ge}$ & 12.01 & 2.63 & 7.05 & 0.84 & 16.59 & 1.50 & 6.97 & 2.13 & 7.10 & 1.54 & 11.74 & 3.80 \\
\hline $\mathrm{Sr}$ & 104.28 & 95.41 & 4.37 & 1.46 & 168.96 & 25.82 & 30.01 & 25.73 & 175.48 & 356.03 & 16.88 & 4.55 \\
\hline $\mathrm{Y}$ & 0.36 & 0.05 & 0.46 & 0.08 & 0.48 & 0.20 & 0.65 & 0.62 & 0.31 & 0.11 & 0.21 & 0.12 \\
\hline $\mathrm{Ta}$ & 1.11 & 0.36 & 1.06 & 0.39 & 1.02 & 0.43 & 1.04 & 1.47 & 0.16 & 0.10 & 0.56 & 0.25 \\
\hline $\mathrm{Pb}$ & 135.44 & 129.63 & 13.36 & 1.50 & 64.72 & 10.31 & 40.34 & 60.43 & 881.33 & 710.51 & 27.57 & 6.20 \\
\hline Th & 0.23 & 0.09 & 1.07 & 0.40 & 0.49 & 0.16 & 1.55 & 1.67 & 0.52 & 0.25 & 0.32 & 0.12 \\
\hline $\mathrm{U}$ & 0.03 & 0.02 & 0.06 & 0.01 & bdl & & 0.06 & 0.02 & 0.06 & 0.06 & 0.02 & 0.02 \\
\hline $\mathrm{La}$ & 22.46 & 4.14 & 23.09 & 7.25 & 33.99 & 10.92 & 18.37 & 19.28 & 9.75 & 8.73 & 14.74 & 5.05 \\
\hline $\mathrm{Ce}$ & 46.61 & 14.32 & 49.63 & 12.21 & 66.11 & 21.77 & 35.44 & 44.29 & 20.35 & 18.36 & 31.63 & 11.11 \\
\hline $\mathrm{Pr}$ & 4.65 & 1.67 & 4.84 & 0.58 & 6.41 & 2.04 & 3.98 & 5.18 & 2.03 & 1.80 & 3.15 & 1.06 \\
\hline $\mathrm{Nd}$ & 12.45 & 5.70 & 11.78 & 0.79 & 18.64 & 5.80 & 13.82 & 16.80 & 7.01 & 4.65 & 8.26 & 2.74 \\
\hline $\mathrm{Sm}$ & 2.83 & 1.40 & 3.86 & 0.57 & 3.76 & 1.18 & 4.74 & 5.45 & 1.73 & 1.19 & 1.91 & 0.70 \\
\hline $\mathrm{Eu}$ & 0.13 & 0.05 & 0.07 & 0.03 & 0.32 & 0.05 & 0.13 & 0.16 & 0.11 & 0.03 & 0.09 & 0.02 \\
\hline $\mathrm{Gd}$ & 0.84 & 0.44 & 1.31 & 0.35 & 1.49 & 0.59 & 1.66 & 1.44 & 0.50 & 0.38 & 0.49 & 0.16 \\
\hline $\mathrm{Tb}$ & 0.08 & 0.02 & 0.15 & 0.02 & 0.17 & 0.02 & 0.21 & 0.18 & 0.07 & 0.05 & 0.07 & 0.03 \\
\hline Dy & 0.24 & 0.12 & 0.48 & 0.17 & 0.41 & 0.14 & 0.61 & 0.61 & 0.21 & 0.10 & 0.16 & 0.05 \\
\hline Ho & bdl & & 0.02 & 0.01 & 0.04 & 0.02 & 0.06 & 0.04 & 0.03 & 0.03 & bdl & \\
\hline $\mathrm{Er}$ & 0.05 & 0.03 & 0.05 & 0.04 & 0.07 & 0.03 & bdl & & 0.05 & 0.02 & 0.08 & 0.04 \\
\hline $\mathrm{Tm}$ & 0.01 & 0.01 & 0.02 & 0.01 & bdl & & 0.03 & 0.01 & 0.02 & bdl & bdl & \\
\hline $\mathrm{Yb}$ & bdl & & bdl & & bdl & & bdl & & 0.15 & 0.20 & 0.10 & 0.01 \\
\hline $\mathrm{Lu}$ & 0.02 & 0.01 & bdl & & bdl & & 0.02 & 0.01 & 0.04 & 0.05 & bdl & \\
\hline$\Sigma$ REE & 90.37 & & 95.29 & & 131.42 & & 79.06 & & 42.06 & & 60.67 & \\
\hline
\end{tabular}

$\mathrm{bdl}=$ below detection limit, $\mathrm{pts}=$ points, $\mathrm{st} \mathrm{dev}=$ standard deviation. 
In general, the concentration of trace elements is variable and does not show any significant trend or correlation with the colour changes. The exceptions are represented by the higher values of $\mathrm{Pb}$ (up to 880 and 2880 ppm in sample 11 and 3b, respectively) corresponding to Mn (Fe)-enriched points and of $\mathrm{Cu}$ (up to $196 \mathrm{ppm}$ ) in samples 1, 4, 9a, determined in the pink part of the crystals. Variable concentration of Ga (41-182 ppm) and Sr (1-231 ppm) was observed. The Y content, generally correlated with HREE, is very low, less than $1 \mathrm{ppm}$ in all samples.

The REE content in tourmalines from granitic pegmatites is generally low ( $<30 \mathrm{ppm}$ ) while in the examined samples, the total REE content ranges from 30 to 130 ppm with light REE-enrichment and Ce being the most abundant element. Chondrite normalized REE patterns (plotted as mean values for each sample in Figure 11) display a general depletion in the medium and heavy, with respect to the light, rare earths. The negative Eu anomaly is probably related to the local depletion of $\mathrm{Eu}^{2+}$ content in the melt due to its consumption during the growth of K-feldspar, an important carrier of Eu ${ }^{2+}$ in magmatic rocks.

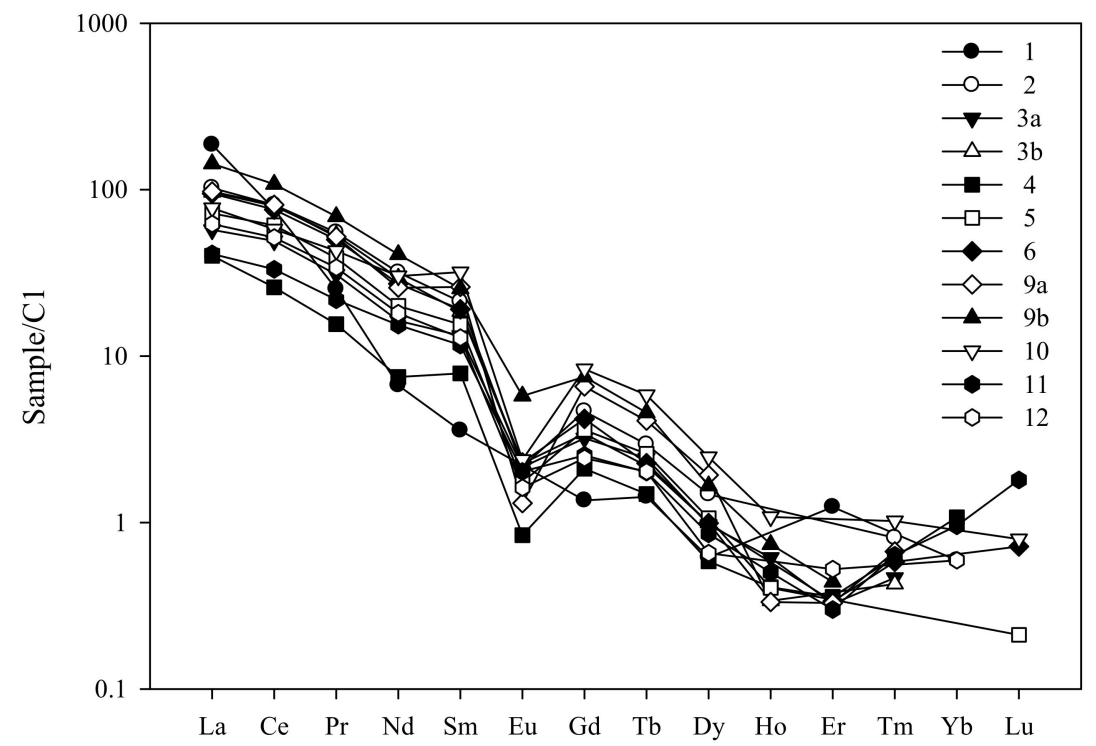

Figure 11. REE patterns normalized to the $C 1$ values reported in [27] for all the analysed samples.

\subsection{X-Ray Crystal Structure Refinement}

The crystallographic data obtained by single crystal refinement confirm that the examined tourmalines belong to the $R 3 m$ space group with the cell parameters in the range of elbaite species. Absolute structure parameter ranged from $0.01(11)$ to $-0.10(10)$ and secondary extinction coefficient ranged from $0.0024(2)$ to 0.0049 (3) (Table 4).

The selected interatomic distances, geometrical parameters and refined and observed (from chemical analyses) site-scattering values are given in Tables 5 and 6, respectively. The final atom coordinates and equivalent displacement parameters, as well as the complete set of crystallographic data (crystallographic information files and lists of observed and calculated structure factors), have been deposited in supplementary electronic material (Tables S1 and S2a-c).

The structural data confirm elbaitic compositions: <Y-O> ranges from 1.989 to $2.030 \AA$ compared to a calculated value of $2.015 \AA$ for an ideal elbaite (using ionic radii of [11]; $<\mathrm{Z}-\mathrm{O}>$ ranges from 1.907 to $1.908 \AA$ compared to a $<^{\mathrm{Z}} \mathrm{Al}-\mathrm{O}>$ grand mean value of $1.906 \AA$ (Figure 3 of [28]); site scattering at $\mathrm{X}$ sites (8.73-12.43 electrons per formula unit or a.p.f.u., Table 6) is compatible with a dominant $\mathrm{Na}$ occupancy. The high quality of the reported structure refinements makes it possible to discuss the site assignment of cations among the different sites of the studied crystals. Following [11] and using his Equation (4) $\left[{ }^{\mathrm{Z}} \mathrm{Al}=-0.1155+1.1713 \cdot{ }^{[6]} \mathrm{Al}-0.0522 \cdot{ }^{[6]} \mathrm{Al}^{2} ; \mathrm{Al}=\mathrm{Al}-{ }^{T} \mathrm{Al}\right.$ (a.p.f.u.) $]$, it is possible to estimate the ${ }^{\mathrm{Z}} \mathrm{Al}$ occupancy just from chemical data. Applying this equation to the data reported 
in Table 2 produces a slight ${ }^{\mathrm{Z}} \mathrm{Al}$ deficiency of $0.126-0.439$ a.p.f.u.. However, this is in contrast with single crystal XRD data that provide refined scattering values of 12.85(5)-12.96(3) a.p.f.u. for the $Z$ site, implying a maximum of $0.12^{\mathrm{Z}} \mathrm{Li}$ atoms per formula unit or just pure $\mathrm{Al} \mathrm{Z}$ sites, considering $3 \sigma$. It is, therefore, highly improbable that any $\mathrm{Mn}^{2+}$ (or $\mathrm{Fe}^{2+}$ ) could have been disordered into the Z-sites. The agreement between observed (SC-XRD) and calculated (EMPA-WDS) site scattering at $X$-sites is poorer (9-10\%, Table 6), probably due to the high chemical variability of crystals, because the occupancy of $X$ site is the main chemical vector observed in the studied tourmalines.

It is worthwhile to note the high values of $U$ equivalent for the $O(1)$ (from 0.0291(7) to 0.057(2) $\AA^{2}$, compared to the mean value of $0.007-0.010 \AA^{2}$ for the other anion sites; see Tables S1 and S2a-c). This is very probably due to static disorder at the $\mathrm{O}(1)$ anion site. All three studied crystals show large and flat [parallel to (0001)] thermal ellipsoids, making the estimation of the electron density at the $\mathrm{O}(1)$ site inaccurate (see Figure 12). We tried a split model, but it was unsuccessful. A similar delocalization of electron density was reported by [29] (compare their Figure 1a with our Figure 12b) for manganese-bearing elbaitic compositions. Burns and co-workers [29] interpreted the large anisotropic displacements as positional disorder, rather than thermal vibration, due to the 6 possible local arrangements at the three $Y$ sites around the $\mathrm{O}(1)$ site; the three principal $Y$ cations ( $\mathrm{Al}, \mathrm{Li}$ and $\mathrm{Mn}^{2+}$ ) have very different ionic radii (0.547(3), 0.751(9), 0.809(1) А, respectively; values from [11]). Considering the composition of the $\mathrm{Y}$ sites (close to AlLiMn) of our tourmalines, the ideal average trimer would promote a distorted environment for the $\mathrm{O}(1)$, confirming in a new set of samples the behaviour already reported by [29].

Table 4. Crystal data and structure refinement for tourmalines 1, 2, 12.

\begin{tabular}{|c|c|c|c|}
\hline Sample & 1 & 2 & 12 \\
\hline Temperature & $293(2) \mathrm{K}$ & 293(2) K & 293(2) K \\
\hline Wavelength & $0.71073 \AA$ & $0.71073 \AA$ & $0.71073 \AA$ \\
\hline Crystal system & Trigonal & Trigonal & Trigonal \\
\hline Space group & $R 3 m$ & $R 3 m$ & $R 3 m$ \\
\hline \multirow[t]{2}{*}{ Unit cell dimensions } & $a=15.8283(3) \AA$ & $a=15.8951(8) \AA$ & $a=15.8909(2) \AA$ \\
\hline & $c=7.09392(18) \AA$ & $c=7.1216(4) \AA$ & $c=7.1163(3) \AA$ \\
\hline Volume & $1539.16(5) \AA^{3}$ & $1558.22(15) \AA^{3}$ & $1556.26(7) \AA^{3}$ \\
\hline Z & 3 & 3 & 3 \\
\hline Absorption coefficient & $1.004 \mathrm{~mm}^{-1}$ & $1.056 \mathrm{~mm}^{-1}$ & $1.020 \mathrm{~mm}^{-1}$ \\
\hline$F(000)$ & 1395 & 1430 & 1422 \\
\hline Crystal size $\left(\mathrm{mm}^{3}\right)$ & $0.29 \times 0.61 \times 0.47$ & $0.35 \times 0.51 \times 0.65$ & $0.40 \times 0.53 \times 0.70$ \\
\hline$\theta$ range for data collection & 3.23 to $36.11^{\circ}$ & 3.22 to $29.04^{\circ}$. & 3.22 to $35.95^{\circ}$ \\
\hline Index ranges & $\begin{array}{c}-26 \leq h \leq 26 \\
-24 \leq k \leq 24 \\
-9 \leq l \leq 9\end{array}$ & $\begin{array}{c}-16 \leq h \leq 13 \\
-20 \leq k \leq 20 \\
-9 \leq l \leq 9\end{array}$ & $\begin{array}{c}-25 \leq h \leq 26 \\
-25 \leq k \leq 25 \\
-9 \leq l \leq 9\end{array}$ \\
\hline Reflections collected & 14,418 & 3806 & 14,500 \\
\hline Independent reflections & 1571 & 914 & 1261 \\
\hline$R$ (int) & 0.0277 & 0.0216 & 0.0334 \\
\hline Completeness to $\theta=35.95^{\circ}$ & $89.30 \%$ & $95.20 \%$ & $72.70 \%$ \\
\hline Refinement method & Full-matrix least-squares on F2 & Full-matrix least-squares on F2 & Full-matrix least-squares on F2 \\
\hline Data/restraints/parameters & $1571 / 1 / 97$ & $914 / 1 / 97$ & $1261 / 1 / 97$ \\
\hline Goodness-of-fit on $F^{2}$ & 1.161 & 1.076 & 1.09 \\
\hline Final $R$ indices $[I>2 \sigma(I)]$ & $\begin{array}{c}R 1=0.0159 \\
\mathrm{w} R 2=0.0400\end{array}$ & $\begin{array}{c}R 1=0.0175 \\
\mathrm{w} R 2=0.0452\end{array}$ & $\begin{array}{c}R 1=0.0179 \\
\mathrm{w} R 2=0.0448\end{array}$ \\
\hline$R$ indices (all data) & $\begin{array}{c}R 1=0.0168 \\
w R 2=0.0403\end{array}$ & $\begin{array}{c}R 1=0.0177 \\
\mathrm{w} R 2=0.0453\end{array}$ & $\begin{array}{c}R 1=0.0186 \\
\mathrm{w} R 2=0.0451\end{array}$ \\
\hline Absolute structure parameter & $0.04(7)$ & $0.01(11)$ & $-0.10(10)$ \\
\hline Extinction coefficient & $0.0049(3)$ & $0.0041(3)$ & $0.0024(2)$ \\
\hline Largest diff. peak and hole & $\begin{array}{c}0.461 \text { and } \\
-0.439 \text { e. } \AA^{-3}\end{array}$ & $\begin{array}{c}0.530 \text { and } \\
-0.497 \text { e. } \AA^{-3}\end{array}$ & $\begin{array}{c}0.889 \text { and } \\
-0.709 \text { e. } \AA^{-3}\end{array}$ \\
\hline
\end{tabular}


Table 5. Bond lengths [^] and angles [ $\left.{ }^{\circ}\right]$ for tourmalines 1, 2, 12.

\begin{tabular}{|c|c|c|c|}
\hline Sample & 1 & 2 & 12 \\
\hline$T-\mathrm{O}(6)$ & $1.6092(9)$ & $1.6049(15)$ & $1.6021(14)$ \\
\hline$T-\mathrm{O}(7)$ & $1.6103(7)$ & $1.6134(13)$ & $1.6110(10)$ \\
\hline $\mathrm{T}-\mathrm{O}(4)$ & $1.6208(5)$ & $1.6263(8)$ & $1.6260(7)$ \\
\hline $\mathrm{T}-\mathrm{O}(5)$ & $1.6363(5)$ & $1.6412(9)$ & $1.6389(8)$ \\
\hline$<T-\mathrm{O}>$ & 1.619 & 1.621 & 1.62 \\
\hline$V\left(\AA^{3}\right)$ & 2.173 & 2.181 & 2.173 \\
\hline TQE & 1.0017 & 1.0023 & 1.0022 \\
\hline TAV & 6.812 & 9.502 & 9.0511 \\
\hline$B-\mathrm{O}(2)$ & $1.3630(18)$ & $1.352(4)$ & $1.361(3)$ \\
\hline$B-\mathrm{O}(8)(\times 2)$ & $1.3798(10)$ & $1.386(2)$ & $1.3850(15)$ \\
\hline$<B-\mathrm{O}>$ & 1.374 & 1.375 & 1.377 \\
\hline$X-\mathrm{O}(2)(\times 3)$ & $2.4570(18)$ & $2.426(3)$ & $2.425(2)$ \\
\hline $\mathrm{X}-\mathrm{O}(5)(\times 3)$ & $2.7422(13)$ & $2.748(2)$ & 2.7492(17) \\
\hline $\mathrm{X}-\mathrm{O}(4)(\times 3)$ & $2.8095(13)$ & $2.810(2)$ & $2.8078(18)$ \\
\hline$<\mathrm{X}-\mathrm{O}>$ & 2.67 & 2.661 & 2.661 \\
\hline$V\left(\AA^{3}\right)$ & 31.14 & 31.419 & 31.086 \\
\hline$Y-\mathrm{O}(2)(\times 2)$ & $1.9620(9)$ & $1.9824(15)$ & $1.9812(14)$ \\
\hline$Y-\mathrm{O}(6)(\times 2)$ & 1.9591(9) & $2.0150(15)$ & $2.0129(13)$ \\
\hline$Y-\mathrm{O}(1)$ & $1.9577(14)$ & $2.028(2)$ & $2.024(2)$ \\
\hline$Y-\mathrm{O}(3)$ & $2.1346(14)$ & $2.171(2)$ & $2.1678(19)$ \\
\hline$\langle\mathrm{Y}-\mathrm{O}\rangle$ & 1.989 & 2.032 & 2.03 \\
\hline$V\left(\AA^{3}\right)$ & 10.125 & 10.796 & 10.759 \\
\hline OQE & 1.0252 & 1.0253 & 1.0253 \\
\hline OAV & 79.39 & 79.54 & 79.7 \\
\hline $\mathrm{Z}-\mathrm{O}(6)$ & $1.8643(8)$ & $1.8506(15)$ & $1.8514(12)$ \\
\hline $\mathrm{Z}-\mathrm{O}(7)$ & $1.8816(8)$ & $1.8823(14)$ & $1.8834(12)$ \\
\hline $\mathrm{Z}-\mathrm{O}(8)$ & $1.8877(8)$ & $1.8865(14)$ & $1.8847(11)$ \\
\hline $\mathrm{Z}-\mathrm{O}(8)$ & $1.9008(8)$ & $1.9114(14)$ & $1.9098(11)$ \\
\hline $\mathrm{Z}-\mathrm{O}(7)$ & $1.9415(7)$ & $1.9551(14)$ & $1.9551(11)$ \\
\hline $\mathrm{Z}-\mathrm{O}(3)$ & $1.9647(6)$ & $1.9617(11)$ & $1.9612(10)$ \\
\hline$<\mathrm{Z}-\mathrm{O}>$ & 1.907 & 1.908 & 1.908 \\
\hline$V\left(\AA^{3}\right)$ & 9.039 & 9.079 & 9.075 \\
\hline OQE & 1.0154 & 1.0137 & 1.0137 \\
\hline OAV & 52.08 & 45.82 & 45.75 \\
\hline $\mathrm{O}(3)-\mathrm{H}(3)$ & $0.80(3)$ & $0.71(4)$ & $0.72(4)$ \\
\hline
\end{tabular}

Table 6. Observed (Single Crystal XRD) and calculated (EMPA) site scattering.

\begin{tabular}{cccc}
\hline \multirow{2}{*}{ Site } & \multicolumn{3}{c}{ (Electrons Per Site, eps) for Tourmalines $\mathbf{1 , 2 , 1 2}$} \\
\cline { 2 - 4 } & $\mathbf{1}$ & $\mathbf{2}$ & $\mathbf{1 2}$ \\
\hline X (obs) & 8.73 & 12.43 & 11.81 \\
X (calc) & 7.95 & 11.34 & 10.61 \\
Y (obs) & 9.49 & 11.98 & 11.26 \\
Y (calc) & 9.25 & 11.97 & 11.12 \\
Z (obs) & 12.96 & 12.83 & 12.85 \\
Z (calc) & 13 & 13 & 13 \\
\hline
\end{tabular}



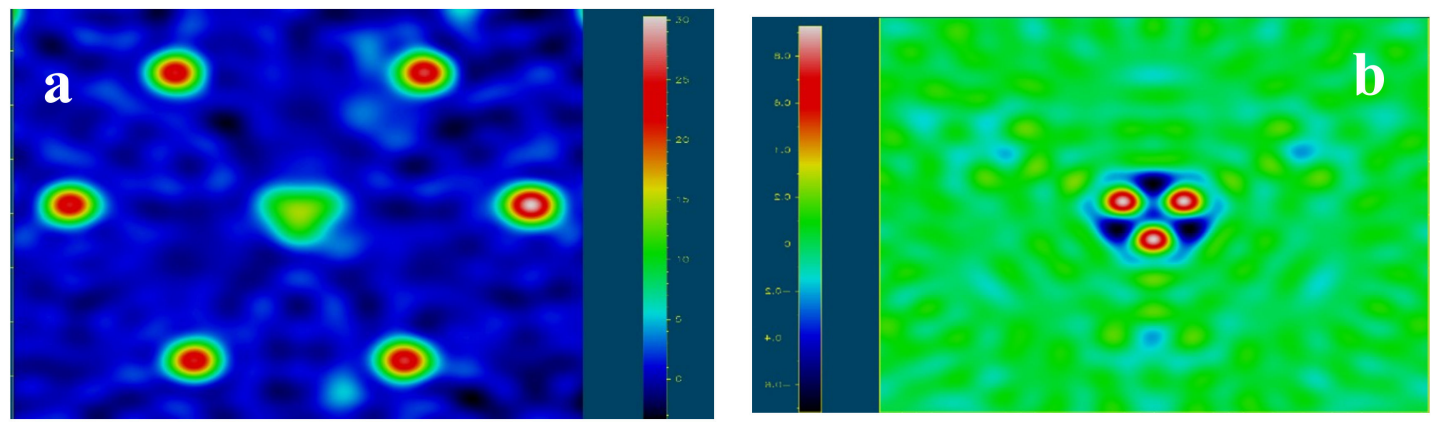

Figure 12. Sample 2: Electron density at $\mathrm{O}(1)$. The high displacement parameters and oblate behaviour of the $\mathrm{O}(1)$ anion site in the three samples are related to the trilobated shape of the maxima at that position. This is ascribable to local static disorder due to the presence of three different size cations as $\mathrm{Al}<\mathrm{Li}<\mathrm{Mn}^{2+}$ in the three $\mathrm{Y}$ coordinating sites for every $\mathrm{O}(1)$ anion site. (a) is a $F_{\mathrm{O}}$ Fourier synthesis; (b) is a $F_{\mathrm{O}}-F_{\mathrm{C}}$ Fourier synthesis.

\section{Discussion and Conclusions}

The tourmalines discovered for the first time in the miarolitic LCT pegmatites at the western border of Adamello Massif may be considered gem materials due to their attractive pink to green-brown hue and transparency. Crystals providing the gem-quality requirements are available, despite the presence of cracks and voids well evidenced by synchrotron X-ray computed micro-tomography.

The studied tourmalines resulted principally in fluor-elbaite, along with minor fluor-liddicoatite, foitite and rossmanite that likely represent the final stages of tourmaline compositional evolution.

The chemical variations, reflecting the environment physicochemical changes during their growth, resulted both in a zoning from nearly black to green to blue to pink elbaite to rossmanite (sample 10) and in a reverse geochemical trend at the latest stages by a pink core, colourless to white midsection and green rim in sections cut perpendicularly to the c-axis (sample 4). In the non-homogeneous samples two different zones, possibly indicating the occurrence of a different and separated generations of tourmaline, can be distinguished: the first can be described as having high $\mathrm{Ca}+\mathrm{Mn}$ or with a continuous fractionation trend of $\mathrm{Mn}$ vs. Fe, and the second one with poor $\mathrm{Ca}+\mathrm{Mn}$ (sample 1) or with a marked enrichment in Fe (sample 4 and 10).

$\mathrm{Mn}$ and $\mathrm{Fe}$ are the main factors controlling the colour of the Li-rich tourmaline. Iron is always lower than manganese, increases in the darker zone and is virtually absent in the near colourless and pink tourmalines. One of the hypotheses on the intensifying pink hues in tourmaline is the presence of manganese in the $3+$ oxidation state. Despite the uranium and thorium contents being very low (6.98 and $0.79 \mathrm{ppm}$ ), the presence, as in sample 9, of uraninite and its natural $\gamma$-radiation could be responsible for the oxidization change from $\mathrm{Mn}^{2+}$ to $\mathrm{Mn}^{3+}$. From our results in tourmalines 1 and 4, it seems that a very low amount ( $<0.08$ a.p.f.u.) of Mn may also be enough to give the bright pink colour. $\mathrm{Cu}^{2+}$ in combination with other cations may modify the resulting colour in tourmalines [1] and, interestingly, the pink parts of samples 1, 4, 9a, corresponding to Ti, Fe and Mn depleted points, contain a relatively high content of $\mathrm{Cu}$.

The REE content is slightly higher than the literature data for tourmalines from relatively primitive NYF (Niobium, Yttrium, Fluorine) and mixed NYF-LCT pegmatites [30]. The chondrite normalized REE patterns are very similar and present a negative $\mathrm{Eu}^{2+}$ anomaly, probably due to the local depletion of $\mathrm{Eu}^{2+}$ in the melt.

The structural data confirm elbaitic compositions of the examined samples. The positional disorder found at the $\mathrm{O}(1)$ anion positions may be due to the high content of manganese entering into the $\mathrm{Y}$ sites together with cations of very different charge and radius ( $\mathrm{Li}$ and $\mathrm{Al})$.

The presence of gem-quality tourmalines in miarolitic cavities suggests the enrichment in volatiles and other exotic elements in pegmatite melt, as well as a shallow level formation in the thermo-metamorphic aureole of the Adamello pluton. The pegmatitic liquids could be exsolved from 
the granitoid magmas during the latest stages of crystallization of the pluton or, alternatively, generated by partial melting of the including metasedimentary sequence.

Further field exploration could turn up additional reserves of gem-quality tourmalines, and their study could contribute to a better understanding of the formation environment of the pegmatitic swarms at the border of Adamello Massif.

Supplementary Materials: The following are available online at http://www.mdpi.com/2075-163X/8/12/ 593/s1, Table S1: Atom coordinates of the three tourmaline crystals of this study, Table S2a: Anisotropic displacement parameters $\left(\AA^{2} \times 10^{3}\right)$ for tourmaline 1. The anisotropic displacement factor exponent takes the form: $-2 \pi^{2}\left[h^{2} a^{* 2} U^{11}+\ldots+2 h k a^{*} b^{*} U^{12}\right]$, Table S2b: Anisotropic displacement parameters $\left(\AA^{2} \times 10^{3}\right)$ for tourmaline 2. The anisotropic displacement factor exponent takes the form: $-2 \pi^{2}\left[h^{2} a^{* 2} U^{11}+\ldots+2 h k a^{*} b^{*}\right.$ $\mathrm{U}^{12}$ ], Table S2c: Anisotropic displacement parameters $\left(\AA^{2} \times 10^{3}\right)$ for tourmaline 12 . The anisotropic displacement factor exponent takes the form: $-2 \pi^{2}\left[h^{2} a^{* 2} U^{11}+\ldots+2 h k a^{*} b^{*} U^{12}\right]$.

Author Contributions: V.D. performed EPMA analyses; F.P. collected the research material; N.M. and G.L. performed the Synchrotron X-ray computed micro-tomography; F.C. performed the SC-XRD analysis; A.L. collected LA-ICP-MS data; I.A. characterized the gemmological materials; V.D., F.P., R.B., N.M., F.C. analysed the data and wrote the paper. All authors contributed to the final version and gave their approval for submission.

Funding: This research received no external funding.

Acknowledgments: We are thankful to Jan Cempirek for fruitful suggestions on electron microprobe data.

Conflicts of Interest: The authors declare no conflict of interest.

\section{References}

1. Pezzotta, F.; Laurs, B.M. Tourmaline: The kaleidoscopic gemstone. Elements 2011, 7, 333-338. [CrossRef]

2. Henry, D.J.; Novák, M.; Hawthorne, F.C.; Ertl, A.; Dutrow, B.L.; Uher, O.; Pezzotta, F. Nomenclature of the tourmaline-supergroup minerals. Am. Mineral. 2011, 96, 895-913. [CrossRef]

3. Pezzotta, F.; Guastoni, A. Adamello: La pegmatite LCT della valle Adamè. Riv. Mineral. Ital. 2002, 3, $127-142$.

4. Mercurio, M.; Rossi, M.; Izzo, F.; Cappelletti, P.; Germinario, C.; Grifa, C.; Petrelli, M.; Vergara, A.; Langella, A. The characterization of natural gemstones using non-invasive FT-IR spectroscopy: New data on tourmalines. Talanta 2018, 178, 147-159. [CrossRef]

5. Van Hinsberg, V.J.; Henry, D.J.; Marschall, H.R. Tourmaline: An ideal indicator of its host environment. Can. Mineral. 2011. [CrossRef]

6. Marinoni, N.; Voltolini, N.; Mancini, L.; Vignola, P.; Pagani, A.; Pavese, A. An investigation of mortars affected by alkali-silica reaction by X-ray synchrotron microtomography: A preliminary study. J. Mater. Sci. 2009, 44, 5815-5823. [CrossRef]

7. Marinoni, N.; Voltolini, N.; Broekmans, M.A.T.M.; Mancini, L.; Monteiro, P.J.M.; Rotiroti, N.; Ferrari, E.; Bernasconi, A. A combined synchrotron radiation micro computed tomography and micro X-ray diffraction study on deleterious alkali-silica reaction. J. Mater. Sci. 2015, 50, 7985-7997. [CrossRef]

8. Hawthorne, F.C.; Henry, D.J. Classification of the minerals of the tourmaline group. Eur. J. Mineral. 1999, 11, 201-215. [CrossRef]

9. Bosi, F.; Andreozzi, G.B.; Skogby, H.; Lussier, A.J.; Abdu, Y.; Hawthorne, F.C. Fluor-elbaite, $\mathrm{Na}\left(\mathrm{Li}_{1.5} \mathrm{Al}_{1.5}\right) \mathrm{Al}_{6}\left(\mathrm{Si}_{6} \mathrm{O}_{18}\right)\left(\mathrm{BO}_{3}\right)_{3}(\mathrm{OH})_{3} \mathrm{~F}$, a new mineral species of the tourmaline supergroup. Am. Mineral. 2013, 98, 297-303. [CrossRef]

10. Bosi, F.; Skogby, H.; Ciriotti, M.E.; Gadas, P.; Novák, M.; Cempírek, J.; Všianský, D.; Filip, J. Lucchesiite, $\mathrm{CaFe}_{3}{ }^{2+} \mathrm{Al}_{6}\left(\mathrm{Si}_{6} \mathrm{O}_{18}\right)\left(\mathrm{BO}_{3}\right)_{3}(\mathrm{OH})_{3} \mathrm{O}$, a new mineral species of the tourmaline supergroup. Miner. Mag. 2017. [CrossRef]

11. Bosi, F. Tourmaline crystal chemistry. Am. Mineral. 2018, 103, 298-306. [CrossRef]

12. Dupuy, C.; Dostal, J.; Fratta, M. Geochemistry of the Adamello Massif (Northern Italy). Contrib. Mineral. Pet. 1982, 80, 41-48. [CrossRef]

13. Zhang, C.; Gieré, R.; Stünitz, H.; Brack, P.; Ulmer, P. Garnet-quartz intergrowths in granitic pegmatites from Bergell and Adamello, Italy. Schweiz. Mineral. Petrogr. Mitt. 2001, 81, 89-113. [CrossRef] 
14. Fiedrich, A.M.; Bachmann, O.; Ulmer, P.; Deering, C.D.; Kunze, K.; Leuthold, J. Mineralogical, geochemical, and textural indicators of crystal accumulation in the Adamello Batholith (Northern Italy). Am. Mineral. 2017, 102, 2467-2483. [CrossRef]

15. Brun, F.; Mancini, L.; Kasae, P.; Favretto, S.; Dreossi, D.; Tromba, G. Pore 3D: A software library for quantitative analysis of porous media. Nucl. Inst. Methods Phys. Res. A 2010, 615, 326-332. [CrossRef]

16. Brun, F.; Massimi, L.; Fratini, M.; Dreossi, D.; Billé, F.; Accardo, A.; Pugliese, R.; Cedola, A. SYRMEP Tomo Project: A graphical user interface for customizing CT reconstruction workflows. Adv. Struct. Chem. Imag. 2017, 3, 1-9. [CrossRef]

17. Herman, G.T. Image Reconstruction from Projections; Academic Press: New York, NY, USA, 1980.

18. Kak, A.C.; Slaney, M. Principles of Computerized Tomographic Imaging; IEEE, Institute for Electrical and Electronic Engineers Press: Piscataway, NY, USA, 1988.

19. Paganin, D.; Mayo, S.C.; Gureyev, T.E.; Miller, P.R.; Wilkins, S.W. Simultaneous phase and amplitude extraction from a single defocused image of a homogeneous object. J. Microsc. 2002, 206, 33-40. [CrossRef]

20. Agilent CrysAlis Computer Program, Agilent Technologies, XRD Products 2012. Available online: http: / / www.agilent.com/chem (accessed on 11 December 2018).

21. Sheldrick, G.M. Crystal structure refinement with SHELXL. Acta Crystallogr. 2015, C71, 3-8.

22. O'Donoghue, M. Gems, 6th ed.; Butterworth-Heinemann: Oxford, UK, 2006.

23. Gübelin, E.J.; Koivula, J.I. Photo Atlas of Inclusions in Gemstones; Opinio Publishers: Basel, Switzerland, 2005; Volume 2.

24. Voltolini, M.; Marinoni, N.; Mancini, L. Synchrotron X-ray computed microtomography investigation of a mortar affected by alkali silica reaction: A quantitative characterization of its microstructural features. J. Mater. Sci. 2011, 46, 6633-6641. [CrossRef]

25. MacDonald, D.J.; Hawthorne, F.C.; Grice, J.D. Foitite, a new alkali deficient tourmaline: Description and crystal structure. Am. Mineral. 1993, 78, 1299-1303.

26. Brown, C.D.; Wise, M.A. Internal zonation and chemical evolution of the Black Mountain granitic pegmatite, Maine. Can. Mineral. 2001, 39, 45-55. [CrossRef]

27. Anders, E.; Grevesse, N. Abundances of the elements: Meteoritic and solar. Geochim. Cosmochim. Acta 1989, 53, 197-214. [CrossRef]

28. Bosi, F.; Andreozzi, G.B. A critical comment on Ertl et al. (2012): “Limitations of $\mathrm{Fe}^{2+}$ and $\mathrm{Mn}^{2+}$ site occupancy in tourmaline: Evidence from $\mathrm{Fe}^{2+}$ - and $\mathrm{Mn}^{2+}$-rich tourmaline". Am. Mineral. 2013, 98, $2183-2192$. [CrossRef]

29. Burns, P.C.; MacDonald, D.J.; Hawthorne, F.C. The crystal-chemistry of manganese-bearing elbaite. Can. Mineral. 1994, 32, 31-41.

30. Čopjaková, R.; Škoda, R.; Vašinová Galiová, M.; Novák, M. Distributions of Y + REE and Sc in tourmaline and their implications for the melt evolution; examples from NYF pegmatites of the Třebíc Pluton, Moldanubian Zone, Czech Republic. J. Geosci. 2013, 58, 113-131. [CrossRef] 